\title{
State-Dependent Regulation of Cortical Processing Speed via Gain Modulation
}

\author{
David Wyrick ${ }^{1}$ and Luca Mazzucato ${ }^{1,2}$ \\ ${ }^{1}$ Department of Biology and Institute of Neuroscience, and ${ }^{2}$ Departments of Mathematics and Physics, University of Oregon, Eugene, Oregon 97403
}

To thrive in dynamic environments, animals must be capable of rapidly and flexibly adapting behavioral responses to a changing context and internal state. Examples of behavioral flexibility include faster stimulus responses when attentive and slower responses when distracted. Contextual or state-dependent modulations may occur early in the cortical hierarchy and may be implemented via top-down projections from corticocortical or neuromodulatory pathways. However, the computational mechanisms mediating the effects of such projections are not known. Here, we introduce a theoretical framework to classify the effects of cell type-specific top-down perturbations on the information processing speed of cortical circuits. Our theory demonstrates that perturbation effects on stimulus processing can be predicted by intrinsic gain modulation, which controls the timescale of the circuit dynamics. Our theory leads to counterintuitive effects, such as improved performance with increased input variance. We tested the model predictions using large-scale electrophysiological recordings from the visual hierarchy in freely running mice, where we found that a decrease in single-cell intrinsic gain during locomotion led to an acceleration of visual processing. Our results establish a novel theory of cell type-specific perturbations, applicable to topdown modulation as well as optogenetic and pharmacological manipulations. Our theory links connectivity, dynamics, and information processing via gain modulation.

Key words: context dependence; gain modulation; locomotion; sensory cortex; spiking networks; visual processing

Significance Statement

To thrive in dynamic environments, animals adapt their behavior to changing circumstances and different internal states. Examples of behavioral flexibility include faster responses to sensory stimuli when attentive and slower responses when distracted. Previous work suggested that contextual modulations may be implemented via top-down inputs to sensory cortex coming from higher brain areas or neuromodulatory pathways. Here, we introduce a theory explaining how the speed at which sensory cortex processes incoming information is adjusted by changes in these top-down projections, which control the timescale of neural activity. We tested our model predictions in freely running mice, revealing that locomotion accelerates visual processing. Our theory is applicable to internal modulation as well as optogenetic and pharmacological manipulations and links circuit connectivity, dynamics, and information processing.

\section{Introduction}

Animals respond to the same stimulus with different reaction times depending on the context or the behavioral state. Faster responses may be elicited by expected stimuli or when the animal is aroused and attentive (Niemi and Näätänen, 1981). Slower responses may occur in the presence of distractors or when the animal is disengaged from the task (Grueninger and Pribram,

\footnotetext{
Received July 20, 2020; revised Mar. 4, 2021; accepted Mar. 8, 2021.

Author contributions: L.M. designed research; L.M. and D.W. performed research; L.M. and D.W. wrote the paper; D.W. analyzed data.

This work was supported by National Institute of Deafness and Other Communication Disorders Grant K25DC013557 and National Institute of Neurological Disorders and Stroke Grant NS118461 (BRAIN Initiative). We thank G. La Camera, Y. Ahmadian, and M. Wehr for useful discussions and suggestions.

The authors declare no competing financial interests.

Correspondence should be addressed to Luca Mazzucato at Imazzuca@uoregon.edu.

https://doi.org/10.1523/JNEUROSCI.1895-20.2021

Copyright ( $\odot 2021$ the authors
}

1969; Treisman and Gelade, 1980; Desimone and Duncan, 1995). Experimental evidence suggests that neural correlates of these contextual modulations occur early in the cortical hierarchy, already at the level of the primary sensory cortex (Jaramillo and Zador, 2011; Samuelsen et al., 2012). During the waking state, levels of arousal, attention, and task engagement vary continuously and are associated with ongoing and large changes in the activity of neuromodulatory systems (Pinto et al., 2013; Fu et al., 2014; A. M. Lee et al., 2014) as well as corticocortical feedback pathways (Nelson et al., 2013; Guo et al., 2014; Zhang et al., 2014; Chen et al., 2017; Leinweber et al., 2017). Activation of these pathways modulates the patterns of activity generated by cortical circuits and may affect their information processing capabilities. However, the precise computational mechanism underlying these flexible reorganizations of cortical dynamics remains elusive.

Variations in behavioral and brain state, such as arousal, engagement, and body movements, may act on a variety of 
timescales, both slow (minutes, hours) and rapid (seconds or subseconds), and spatial scales, both global (pupil diameter, orofacial movements) and brain subregion-specific; and they can be recapitulated by artificial perturbations, such as optogenetic, chemogenetic, or electrical stimulation. These variations have been associated with a large variety of seemingly unrelated mechanisms operating both at the single-cell and at the population level. At the population level, these mechanisms include modulations of low- and high-frequency rhythmic cortical activities (McGinley et al., 2015), changes in noise correlations (Cohen and Maunsell, 2009; Dadarlat and Stryker, 2017), and increased information flow between cortical and subcortical networks (McGinley et al., 2015). On a cellular level, these variations have been associated with modulations of single-cell responsiveness and reliability (Dadarlat and Stryker, 2017), and cell type-specific gain modulation (McGinley et al., 2015). These rapid, trial-bytrial modulations of neural activity may be mediated by neuromodulatory pathways, such as cholinergic and noradrenergic systems (Pinto et al., 2013; Fu et al., 2014; A. M. Lee et al., 2014; Reimer et al., 2016), or more precise corticocortical projections from prefrontal areas toward primary sensory areas (Nelson et al., 2013; Guo et al., 2014; Zhang et al., 2014; Chen et al., 2017; Leinweber et al., 2017). The effects of these corticocortical projections can be recapitulated by optogenetic activation of glutamatergic feedback pathways (Zagha et al., 2015). In the face of this wide variety of physiological pathways, is there a common computational principle underlying the effects they elicit on sensory cortical circuits?

A natural way to model the effect of activating a specific pathway on a downstream circuit is in the form of a perturbation to the downstream circuit's afferent inputs or recurrent couplings (Huang et al., 2019; Mazzucato et al., 2019). Here, we will present a theory explaining how these perturbations control the information processing speed of a downstream cortical circuit. Our theory shows that the effects of perturbations that change the statistics of the afferents or the recurrent couplings can all be captured by a single mechanism of action: intrinsic gain modulation, where gain is defined as the rate of change of the intrinsic input/output transfer function of a neuron measured during periods of ongoing activity. Our theory is based on a biologically plausible model of cortical circuits using clustered spiking network (Amit and Brunel, 1997). This class of models capture complex physiological properties of cortical dynamics, such as state-dependent changes in neural activity, variability (Deco and Hugues, 2012; Litwin-Kumar and Doiron, 2012; Mazzucato et al., 2015, 2016; Rostami et al., 2020), and information processing speed (Mazzucato et al., 2019). Our theory predicts that gain modulation controls the intrinsic temporal dynamics of the cortical circuit; thus, its information processing speed, such as that decreasing the intrinsic single-cell gain, leads to faster stimulus coding.

We tested our theory by examining the effect of locomotion on visual processing in the visual hierarchy. We found that locomotion decreased the intrinsic gain of visual cortical neurons in the absence of stimuli in freely running mice. The theory thus predicted a faster encoding of visual stimuli during running compared with rest, which we confirmed in the empirical data. Our theoretical framework links gain modulation to information processing speed, providing guidance for the design and interpretation of future manipulation experiments by unifying the changes in brain state because of behavior, optogenetic, or pharmacological perturbations, under the same shared mechanism.
Table 1. Parameters for the clustered network used in the simulations

\begin{tabular}{llc}
\hline Parameter & Description & Value \\
\hline$j_{E E}$ & Mean E-to-E synaptic weights $\times \sqrt{N}$ & $0.6 \mathrm{mV}$ \\
$j_{I E}$ & Mean E-to-I synaptic weights $\times \sqrt{N}$ & $0.6 \mathrm{mV}$ \\
$j_{E I}$ & Mean I-to-E synaptic weights $\times \sqrt{N}$ & $1.9 \mathrm{mV}$ \\
$j_{I I}$ & Mean I-to-I synaptic weights $\times \sqrt{N}$ & $3.8 \mathrm{mV}$ \\
$j_{E 0}$ & Mean E-to-E synaptic weights $\times \sqrt{N}$ & $2.6 \mathrm{mV}$ \\
$j_{I 0}$ & Mean I-to-I synaptic weights $\times \sqrt{N}$ & $2.3 \mathrm{mV}$ \\
$\delta$ & SD of the synaptic weight distribution & $20 \%$ \\
$J_{E E}^{+}$ & Potentiated intracluster E-to-E weight factor & 14 \\
$J_{I I}^{+}$ & Potentiated intracluster I-to-I weight factor & 5 \\
$g_{E I}$ & Potentiation parameter for intracluster I-to-E weights & 10 \\
$g_{I E}$ & Potentiation parameter for intracluster E-to-I weights & 8 \\
$r_{\text {ext }}$ & Average baseline afferent rate to E and I neurons & $5 \mathrm{spks} / \mathrm{s}$ \\
$V_{E}^{\text {thr }}$ & E-neuron threshold potential & $1.43 \mathrm{mV}$ \\
$V_{I}^{\text {thr }}$ & I-neuron threshold potential & $0.74 \mathrm{mV}$ \\
$V^{\text {reset }}$ & E- and I-neuron reset potential & $0 \mathrm{mV}$ \\
$\tau_{m}$ & E- and I-neuron membrane time constant & $20 \mathrm{~ms}$ \\
$\tau_{\text {refr }}$ & E- and I-neuron absolute refractory period & $5 \mathrm{~ms}$ \\
$\tau_{s}$ & E- and I-neuron synaptic time constant & $5 \mathrm{~ms}$ \\
\hline
\end{tabular}

\section{Materials and Methods}

Spiking network model

Architecture. We modeled the local cortical circuit as a network of $N=2000$ excitatory (E) and inhibitory (I) neurons (with relative fraction $n_{E}=80 \%$ and $n_{I}=20 \%$ ) with random recurrent connectivity (see Fig. 2). Connection probabilities were $p_{E E}=0.2$ and $p_{E I}=p_{I E}=p_{I I}=0.5$. Nonzero synaptic weights from presynaptic neuron $j$ to postsynaptic neuron $i$ were $J_{i j}=j_{i j} / \sqrt{N}$, with $j_{i j}$ sampled from a Gaussian distribution with mean $j_{\alpha \beta}$, for $\alpha, \beta=E, I$, and SD $\delta^{2}$. E and I neurons were arranged in $p$ clusters. E clusters had heterogeneous sizes drawn from a Gaussian distribution with a mean of $N_{E}^{\text {clust }}=80$ E-neurons and $20 \%$ SD. The number of clusters was then determined as $p=$ round $\left(n_{E} N\left(1-n_{b g r}\right) / N_{E}^{\text {clust }}\right)$, where $n_{b g r}=0.1$ is the fraction of background neurons in each population (i.e., not belonging to any cluster). I clusters had equal size $N_{I}^{\text {clust }}=\operatorname{round}\left(n_{I} N\left(1-n_{\text {bgr }} / p\right)\right.$. Clusters were defined by an increase in intracluster weights and a decrease in intercluster weights, under the constraint that the net input current to a neuron would remain unchanged compared with the case without clusters. Synaptic weights for within cluster neurons where potentiated by a ratio factor $J_{\alpha \beta}^{+}$. Synaptic weights between neurons belonging to different clusters were depressed by a factor $J_{\alpha \beta}^{-}$. Specifically, we chose the following scaling: $J_{E I}^{+}=p /\left(1+(p-1) / g_{E I}\right), J_{I E}^{+}=p /\left(1+(p-1) / g_{I E}\right), J_{E I}^{-}=J_{E I}^{+} /$ $g_{E I}, J_{I E}^{-}=J_{I E}^{+} / g_{I E}$ and $J_{\alpha \alpha}^{-}=1-\gamma\left(J_{\alpha \alpha}^{+}-1\right)$ for $\alpha=E$, $I$, with $\gamma=f(2-f(p+1))^{-1}$, where $f=\left(1-n_{b g r}\right) / p$ is the fraction of E neurons in each cluster. Within cluster E-to-E synaptic weights were further multiplied by cluster-specific factor equal to the ratio between the average cluster size $N_{E}^{\text {clust }}$ and the size of each cluster, so that larger clusters had smaller within cluster couplings. We chose network parameters so that the cluster timescale was $100 \mathrm{~ms}$, as observed in cortical circuits (Jones et al., 2007; Mazzucato et al., 2015, 2019). Parameter values are in Table 1.

Neuronal dynamics. We modeled spiking neurons as current-based leaky-integrate-and-fire (LIF) neurons whose membrane potential $V$ evolved according to the following dynamical equation:

$$
\frac{d V}{d t}=-\frac{V}{\tau_{m}}+I_{r e c}+I_{e x t}
$$

where $\tau_{m}$ is the membrane time constant. Input currents included a contribution $I_{r e c}$ coming from the other recurrently connected neurons in the local circuit and an external current $I_{\text {ext }}=I_{0}+I_{\text {stim }}+I_{\text {pert }}$ (units of $\mathrm{mV} \mathrm{s}^{-1}$ ). The first term $I_{0}=N_{e x t} J_{\alpha 0} r_{\text {ext }}$ (for $\alpha=E, I$ ) is a constant term representing input to the $\mathrm{E}$ or I neuron from other brain areas and $N_{\text {ext }}=n_{E} N p_{E E}$, whereas $I_{\text {stim }}$ and $I_{\text {pert }}$ represent the 


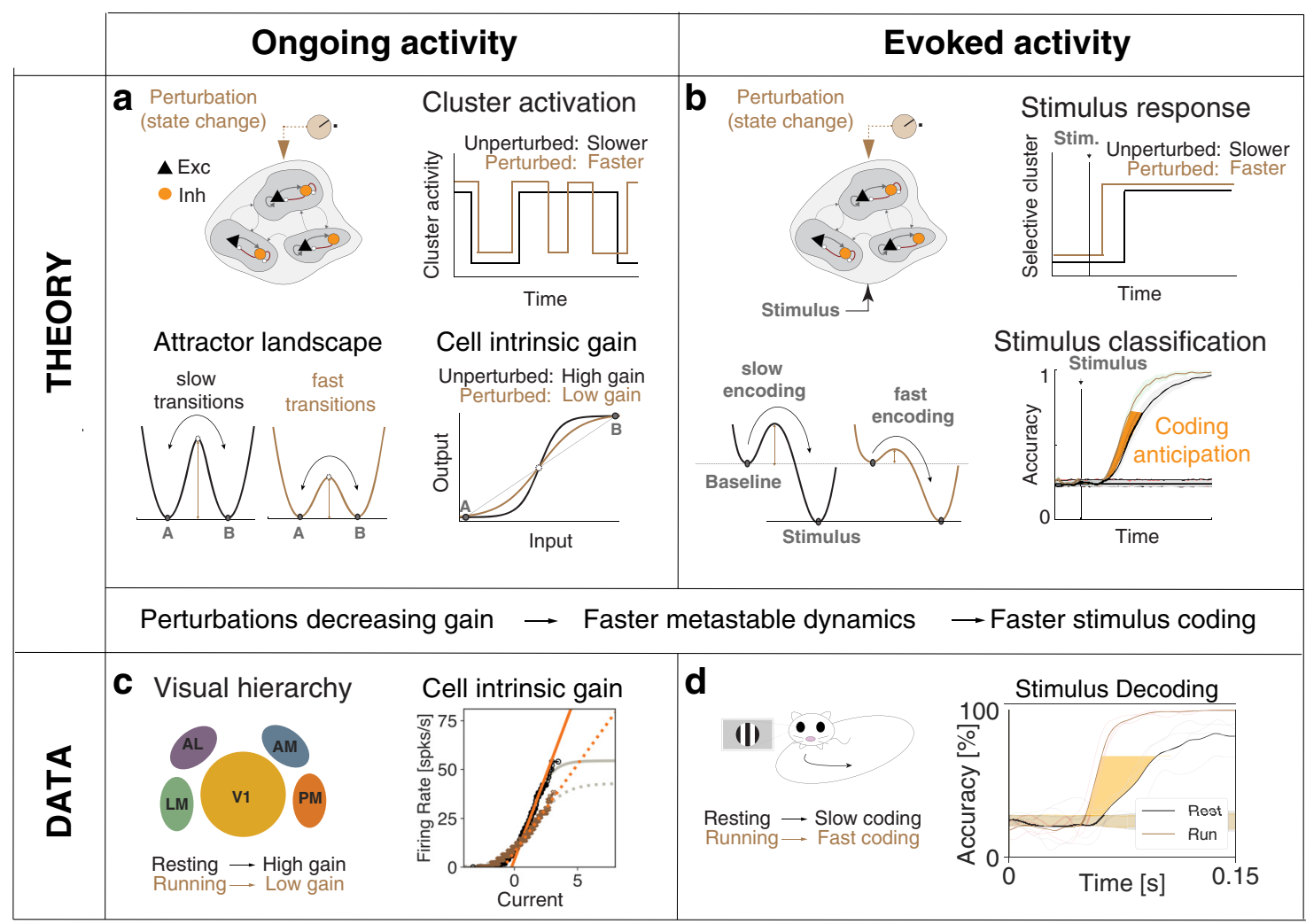

Figure 1. Conceptual summary of the main results. $\boldsymbol{a}$, In a network model of sensory cortex featuring clusters of excitatory and inhibitory neurons with metastable dynamics, state changes are induced by external perturbations controlling the timescale of cluster activation during ongoing activity. The neural mechanism underlying timescale modulation is a change in the barrier height separating attractors, driven by a modulation of the intrinsic gain of the single-cell transfer function. $\boldsymbol{b}$, During evoked activity, onset of stimulus encoding is determined by the activation latency of stimulus-selective cluster. External perturbations modulate the onset latency, thus controlling the stimulus processing speed. The theory shows that the effect of external perturbations on stimulus processing speed during evoked activity (right) can be predicted by the induced gain modulations observed during ongoing activity (left). $c$, Locomotion induced changes in intrinsic gain in the visual cortical hierarchy during darkness periods. $\boldsymbol{d}$, Locomotion drove faster coding of visual stimuli during evoked periods, as predicted by the induced gain modulations observed during ongoing activity.

incoming sensory stimulus or the various types of perturbation (see Stimuli and perturbations below). When $V$ hits threshold $V_{\alpha}^{t h r}($ for $\alpha=E, I$ ), a spike is emitted and $V$ is then held at the reset value $V^{\text {reset }}$ for a refractory period $\tau_{\text {refr }}$. We chose the thresholds so that the homogeneous network (i.e., where all $J_{\alpha \beta}^{ \pm}=1$ ) was in a balanced state with average spiking activity at rates $\left(r_{E}, r_{I}\right)=(2,5)$ spks/s (Amit and Brunel, 1997; Mazzucato et al., 2019). Postsynaptic currents evolved according to the following equation:

$$
\tau_{s y n} \frac{d I_{r e c}}{d t}=-I_{r e c}+\sum_{j=1}^{N} J_{i j} \sum_{k} \delta\left(t-t_{k}\right)
$$

where $\tau_{s}$ is the synaptic time constant, $J_{i j}$ are the recurrent couplings, and $t_{k}$ is the time of the $\mathrm{k}$-th spike from the $\mathrm{j}$-th presynaptic neuron. Parameter values are in Table 1.

Sensory stimuli. We considered two classes of inputs: sensory stimuli and perturbations. In the "evoked" condition (see Fig. 4a), we presented the network one of four sensory stimuli, modeled as changes in the afferent currents targeting $50 \%$ of E-neurons in stimulus-selective clusters; each E cluster had a $50 \%$ probability of being selective to a sensory stimulus (mixed selectivity). In the first part of the paper (Fig. 1; see Figs. 2-6), I clusters were not stimulus-selective. Moreover, in both the unperturbed and the perturbed stimulus-evoked conditions, stimulus onset occurred at time $t=0$ and each stimulus was represented by an afferent current $I_{\text {stim }}(t)=I_{\text {ext }} r_{\text {stim }}(t)$, where $r_{\text {stim }}(t)$ is a linearly ramping increase reaching a value $r_{\text {max }}=20 \%$ above baseline at $t=1$. In the last part of the paper (see Fig. 7), we introduced a new stimulation protocol where visual stimuli targeted both $\mathrm{E}$ and I clusters in pairs, corresponding to thalamic input onto both excitatory and inhibitory neurons in V1 (Zhuang et al., 2013; Kloc and Maffei, 2014; Khan et al., 2018; Miska et al., 2018). Each E-I cluster pair had a 50\% probability of being selective to each visual stimulus. If a E-I cluster pair was selective to a stimulus, then all E neurons and $50 \%$ of I neurons in that pair received the stimulus. The time course of visual stimuli was modeled as a double exponential profiles with rise and decay times of $(0.05,0.5 \mathrm{~s})$, and peak equal to a $20 \%$ increase compared with the baseline external current.

External perturbations. We considered several kinds of perturbations. In the perturbed stimulus-evoked condition (see Fig. 4b), perturbation onset occurred at time $t=-0.5$ and lasted until the end of the stimulus presentation at $t=1$ with a constant time course. We also presented perturbations in the absence of sensory stimuli ("ongoing" condition; see Figs. 2, 3); in that condition, the perturbation was constant and lasted for the whole duration of the trial ( $5 \mathrm{~s})$. Finally, when assessing single-cell responses to perturbations, we modeled the perturbation time course as a double exponential with rise and decay times $[0.1,1 \mathrm{~s}]$ (see Fig. 6). In all conditions, perturbations were defined as follows:

- $\delta$ mean(E), $\delta$ mean(I): A constant offset $I_{\text {pert }}=z I_{0}$ in the mean afferent currents was added to all neurons in either $\mathrm{E}$ or I populations, respectively, expressed as a fraction of the baseline value $I_{0}$ (see Neuronal dynamics above), where $z \in[-0.1,0.2]$ for E neurons and $z$ $\in[-0.2,0.2]$ for I neurons.

- $\delta \operatorname{var}(\mathrm{E}), \delta \operatorname{var}(\mathrm{I}):$ For each $\mathrm{E}$ or I neuron, respectively, the perturbation was a constant offset $I_{\text {pert }}=z I_{0}$, where $z$ is a gaussian random variable with zero mean and SD $\sigma$. We chose $\sigma \in[0,0.2]$ for E neurons and $\sigma \in[0,0.5]$ for I neurons. This perturbation did not change 
the mean afferent current but only its spatial variance across the $\mathrm{E}$ or I population, respectively. We measured the strength of these perturbations via their coefficient of variability $C V(\alpha)=\sigma_{\alpha} / \mu_{\alpha}$, for $\alpha=E$, $I$, where $\sigma$ and $\mu=I_{0}$ are the SD and mean of the across-neuron distribution of afferent currents.

- $\delta$ AMPA: A constant change in the mean $j_{\alpha E} \rightarrow(1+z) j_{\alpha E}$ synaptic couplings (for $\alpha=E, I$ ), representing a modulation of glutamatergic synapses. We chose $z \in[-0.1,0.2]$.

- $\delta$ GABA: A constant change in the mean $j_{\alpha I} \rightarrow(1+z) j_{\alpha I}$ synaptic couplings (for $\alpha=E, I$ ), representing a modulation of GABAergic synapses. We chose $z \in[-0.2,0.2]$.

The range of the perturbations were chosen so that the network still produced metastable dynamics for all values.

Inhibition stabilization. We simulated a stimulation protocol used in experiments to test inhibition stabilization (see Fig. $2 c$ ). This protocol is identical to the $\delta$ mean(I) perturbation during ongoing periods, where the perturbation targeted all I neurons with an external current $I_{\text {pert }}=z I_{0}$ applied for the whole length of $5 \mathrm{~s}$ intervals, with $z \in[0,1.2]$ and 40 trials per network and 10 networks for each value of the perturbation.

Simulations. All data analyses, model simulations, and mean field theory calculations were performed using custom software written in MATLAB, C, and Python. Simulations in the stimulusevoked conditions (both perturbed and unperturbed) comprised 10 realizations of each network (each network with different realization of synaptic weights), with 20 trials for each of the four stimuli. Simulations in the ongoing condition comprised 10 different realizations of each network, with 40 trials per perturbation. Each network was initialized with random synaptic weights and simulated with random initial conditions in each trial. Sample sizes were similar to those reported in previous publications (Mazzucato et al., 2015, 2016, 2019). Dynamical equations for the LIF neurons were integrated with the Euler method with a $0.1 \mathrm{~ms}$ step. Code to simulate the model with $\delta \operatorname{var}(\mathrm{E})$ perturbation and to perform the decoding analysis on the Allen Institute Neuropixel dataset has been uploaded to https:/github.com/ mazzulab/cortical_processing_speed. Code to reproduce the full set of perturbations investigated in this paper is available on request to the corresponding author.

\section{Mean field theory}

We performed a mean field analysis of a simplified two cluster network for LIF neurons with exponential synapses, comprising $p+2$ populations for $p=2$ (Amit and Brunel, 1997; Mazzucato et al., 2019): the first $p$ represents the two $\mathrm{E}$ clusters, the last two represent the background $\mathrm{E}$ and the I population. The infinitesimal mean $\mu_{n}$ and variance $\sigma_{n}^{2}$ of the postsynaptic currents are as follows:

$$
\begin{aligned}
& \mu_{n}=\tau_{m} \sqrt{N}\left[n_{E} p_{E E} j_{E E}\left(f J_{E E}^{+} r_{n}+J_{E E}^{-}\left(\sum_{l=1}^{p-1} r_{l}+(1-p f) r_{E}^{b g r}\right)+\frac{j_{E 0}}{j_{E E}} r_{e x t}\right)-n_{I} p_{E I} j_{E I} r_{I}\right], \\
& \mu_{b g r}=\tau_{m} \sqrt{N}\left[n_{E} p_{E E} j_{E E}\left(J_{E E}^{-} \sum_{l=1}^{p} r_{l}+(1-p f) r_{E}^{b g r}+\frac{j_{E 0}}{j_{E E}} r_{e x t}\right)-n_{I} p_{E I} j_{E I} r_{I}\right], \\
& \mu_{I}=\tau_{m} \sqrt{N}\left[n_{E} p_{I E} j_{I E}\left(f \sum_{l=1}^{p} r_{l}+(1-p f) r_{E}^{b g r}\right)-n_{I} p_{I I}\left(j_{I I} r_{I}+j_{I 0} r_{e x t}\right)\right],
\end{aligned}
$$

$$
\begin{aligned}
& \left.\sigma_{n}^{2}=\tau_{m} \sqrt{N}\left[n_{E} p_{E E} j_{E E}^{2}\left(f\left(J_{E E}^{+}\right)^{2} r_{n}+\left(J_{E E}^{-}\right)^{2}\left(\sum_{l=1}^{p-1} r_{l}+(1-p f) r_{E}^{b g r}\right)\right)\right)-n_{I} p_{E j} j_{E I}^{2} r_{I}\right], \\
& \sigma_{b g r}^{2}=\tau_{m} \sqrt{N}\left[n_{E} p_{E E} j_{E E}^{2}\left(\left(J_{E E}^{-}\right)^{2} \sum_{l=1}^{p} r_{l}+(1-p f) r_{E}^{b g r}\right)-n_{I} p_{E I} j_{E I}^{2} r_{I}\right], \\
& \sigma_{I}^{2}=\tau_{m} \sqrt{N}\left[n_{E} p_{I E} j_{I E}^{2}\left(f \sum_{l=1}^{p} r_{l}+(1-p f) r_{E}^{b g r}\right)-n_{I} p_{I I} j_{I I}^{2} r_{I}\right],
\end{aligned}
$$

where $r_{n}, r_{l}=1, \ldots, p$ are the firing rates in the $p$ E clusters; $r_{E}^{b r}, r_{I}, r_{\text {ext }}$ are the firing rates in the background $\mathrm{E}$ population, in the I population, and in the external current. Other parameters are described in Architecture and in Table 2. This simplified network has fixed in-degree determined by $\$ p_{-}\{\alpha \beta\} \$$ for $\$ \alpha, \beta=\mathrm{E}$, I\$. The network attractors satisfy the self-consistent fixed-point equations as follows:

$$
r_{l}=F_{l}\left[\mu_{l}(\mathbf{r}), \sigma_{l}^{2}(\mathbf{r})\right],
$$

where $\mathbf{r}=\left(r_{1}, \ldots, r_{p}, r_{b g r}, r_{I}\right)$ and $l=1, \ldots, p, b g r, I$, and $F_{l}$ is the currentto-rate transfer function for each population, which depend on the condition. In the absence of perturbations, all populations have the LIF transfer function as follows:

$$
F_{l}\left(\mu_{l}, \sigma_{l}\right)=\left(\tau_{\text {refr }}+\tau_{m} \sqrt{\pi} \int_{H_{l}}^{\Theta_{l}} e^{u^{2}}[1+\operatorname{erf}(u)]\right)^{-1},
$$

where $H_{l}=\left(V^{\text {reset }}-\mu_{l}\right) / \sigma_{l}+a k$ and $\Theta_{l}=\left(V_{l}^{\text {thr }}-\mu_{l}\right) / \sigma_{l}+a k$. $k=\sqrt{\tau_{s} / \tau_{m}}$ and $a=|\zeta(1 / 2)| / \sqrt{2}$ are terms accounting for the synaptic dynamics (Fourcaud and Brunel, 2002). The perturbations $\delta \operatorname{var}(\mathrm{E})$ and $\delta \operatorname{var}(\mathrm{I})$ induced an effective population transfer function $F^{\text {eff }}$ on the E and I populations (Mazzucato et al., 2019), respectively, given by the following:

$$
F_{\alpha}^{\text {pert }}\left(\mu_{\alpha}, \sigma_{\alpha}\right)=\int D z F_{\alpha}\left(\mu_{\alpha}+z \sigma_{z} \mu_{\alpha}^{e x t}, \sigma_{\alpha}^{2}\right),
$$

where $\alpha=E, I$ and $D z=d z \exp \left(-z^{2} / 2 / \sqrt{2 \pi}\right)$ is a Gaussian measure of zero mean and unit variance, $\mu_{\alpha}^{e x t}=\tau_{m} \sqrt{N} n_{\alpha} p_{\alpha 0} j_{\alpha 0} r_{\text {ext }}$ is the external current and $\sigma_{z}$ is the SD of the perturbation with respect to baseline, denoted CV(E) and CV(I). Stability of the fixed-point Equation 1.3 was defined with respect to the approximate linearized dynamics of the instantaneous mean $m_{l}$ and variance $s_{l}^{2}$ of the input currents (Mazzucato et al., 2015, 2019) as follows:

$$
\begin{gathered}
\tau_{s} \frac{d m_{l}}{d t}=-m_{l}+\mu_{l}\left(r_{l}\right) ; \quad \tau_{s} \frac{d s_{l}^{2}}{2 d t}=-s_{l}^{2}+\sigma_{l}^{2}\left(r_{l}\right) ; \\
r_{l}=F_{l}\left(m_{l}(\mathbf{r}), s_{l}^{2}(\mathbf{r})\right)
\end{gathered}
$$

where $\mu_{l}, \sigma_{l}^{2}$ (for $1=$ E,bgr,I) are defined in Equations 1.1 and 1.2, and $F_{l}$ represents the appropriate transfer function 1.4 or 1.5. Fixed-point stability required that the stability matrix as follows:

$$
S_{l m}=\frac{1}{\tau_{s}}\left(\frac{\partial F_{l}\left(\mu_{l}, \sigma_{l}^{2}\right)}{\partial r_{m}}-\frac{\partial F_{l}\left(\mu_{l}, \sigma_{l}^{2}\right)}{\partial \sigma_{l}^{2}} \frac{\partial \sigma_{l}^{2}(\mathbf{r})}{\partial r_{m}}-\delta_{l m}\right),
$$

was negative definite. The full mean field theory described above was used for the comprehensive analysis of Figure 3. For the schematic of Figure $3 c$, we replaced the LIF transfer function 1.4 with the simpler function $\tilde{F}\left(\mu_{E}\right)=0.5\left(1+\tanh \left(\mu_{E}\right)\right)$ and the $\delta \operatorname{var}(\mathrm{E})$ perturbation effect was then modeled as $\tilde{F}^{\text {eff }}(\mu)=\int D z \tilde{F}\left(\mu_{E}+z \sigma_{z} \mu_{\text {ext }}\right)$.

Effective mean field theory for a reduced network. To calculate the potential energy barrier separating the two network attractors in the reduced two cluster network, we used the effective mean field theory developed previously (Mascaro and Amit, 1999; Mattia et al., 2013; Mazzucato et al., 2019). The idea is to first estimate the force acting on neural configurations with cluster firing rates $\mathbf{r}=\left[\tilde{r}_{1}, \tilde{r}_{2}\right]$ outside the fixed points (Eq. 1.3), then project the two-dimensional system onto a one-dimensional trajectory along which the force can be integrated to give an effective potential $E$. In the first step, we start from the full mean field equations for the $P=p+2$ populations in Equation 1.3, and obtain an effective description of the dynamics for $q$ populations "in focus" describing E clusters ( $q=2$ in our case) by integrating out the remaining $P-q$ out-of-focus populations describing the background $\mathrm{E}$ neurons and the I neurons $(P-q=2$ in our case). Given a fixed value $\tilde{\mathbf{r}}=\left[\tilde{r}_{1}, \ldots, \tilde{r}_{q}\right]$ for the $q$ in-focus populations, one obtains 
the stable fixed-point firing rates $\mathbf{r}^{\prime}=\left[r_{q+1}^{\prime}, \ldots, r_{P}^{\prime}\right]$ of the out-of-focus populations by solving their mean field equations as follows:

$$
r_{\beta}^{\prime}(\tilde{\mathbf{r}})=F_{\beta}\left[\mu_{\beta}\left(\tilde{\mathbf{r}}, \mathbf{r}^{\prime}\right), \sigma_{\beta}^{2}\left(\tilde{\mathbf{r}}, \mathbf{r}^{\prime}\right)\right]
$$

for $\beta=q+1, \ldots, P$, as function of the infocus populations $\tilde{\mathbf{r}}$, where stability is calculated with respect to the condition (Eq. 1.7) for the reduced $(q+1, \ldots, P)$ out-of-focus populations at fixed values of the in-focus rates $\tilde{\mathbf{r}}$. One then obtains a relation between the input $\tilde{\mathbf{r}}$ and output values $\tilde{\mathbf{r}}^{\text {out }}$ of the infocus populations by inserting the fixed-point rates of the out-of-focus populations (calculated in Eq. 1.8) as follows:

$$
r_{\alpha}^{\text {out }}(\tilde{\mathbf{r}})=F_{\alpha}\left[\mu_{\alpha}\left(\tilde{\mathbf{r}}, \mathbf{r}^{\prime}(\tilde{\mathbf{r}})\right), \sigma_{\alpha}^{2}\left(\tilde{\mathbf{r}}, \mathbf{r}^{\prime}(\tilde{\mathbf{r}})\right)\right],
$$

for $\alpha=1, \ldots, q$. The original fixed points are $\tilde{\mathbf{r}}^{*}$ such that $\tilde{r}_{\alpha}^{*}=r_{\alpha}^{\text {out }}\left(\tilde{\mathbf{r}}^{*}\right)$.

Potential energy barriers and transfer function gain. In a reduced network with two in-focus populations $\left[\tilde{r}_{1}, \tilde{r}_{2}\right]$ corresponding to the two E clusters, one can visualize Equation 1.9 as a two-dimensional force vector $\tilde{\mathbf{r}}-\mathbf{r}^{\text {out }}(\tilde{\mathbf{r}})$ at each point in the two-dimensional firing rate space $\tilde{\mathbf{r}}$. The force vanishes at the stable fixed points $A$ and $B$ and at the unstable fixed-point $C$ between them (see Fig. $3 c$ ). One can further reduce the system to one dimension by approximating its dynamics along the trajectory between $A$ and $B$ as in Mascaro and Amit (1999) as follows:

$$
\tau_{s} \frac{d \tilde{r}}{d t}=-\tilde{r}+r^{\text {out }}(\tilde{r})
$$

where $y=r^{\text {out }}(\tilde{r})$ represents an effective transfer function and $\tilde{r}-r^{\text {out }}(\tilde{r})$ an effective force. We estimated the gain $g$ of the effective transfer function as $g=1-\frac{r^{\text {out }}\left(\tilde{r}_{\text {min }}\right)-r^{\text {out }}\left(\tilde{r}_{\text {min }}\right)}{\tilde{r}_{\text {min }}-\tilde{r}_{\text {max }}}$, where $\tilde{r}_{\text {min }}$ and $\tilde{r}_{\max }$ represent, respectively, the minimum and maximum of the force (see Fig. $3 c$ ). From the one-dimensional dynamics (Eq. $1.10)$, one can define a potential energy via $\frac{\partial E(\tilde{r})}{\partial r}=\tilde{r}-r^{\text {out }}(\tilde{r})$. The energy minima represent the stable fixed points $A$ and $B$ and the saddle point $C$ between them represents the potential energy barrier separating the two attractors. The height $\Delta$ of the potential energy barrier is then given by the following:

$$
\Delta=\int_{A}^{C} d \tilde{r}\left[\tilde{r}-r^{\text {out }}(\tilde{r})\right]
$$

which can be visualized as the area of the curve between the effective transfer function and the diagonal line (see Fig. 3).

\section{Experimental data}

We tested our model predictions using the open-source dataset of neuropixel recordings from the Allen Institute for Brain Science (Siegle et al., 2019). We focused our analysis on experiments where drifting a Network architecture

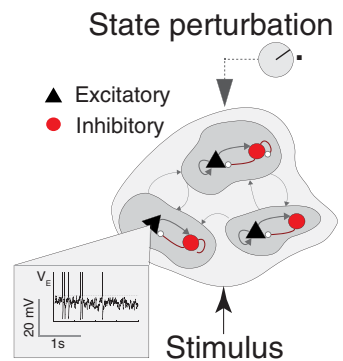

b

Ongoing activity

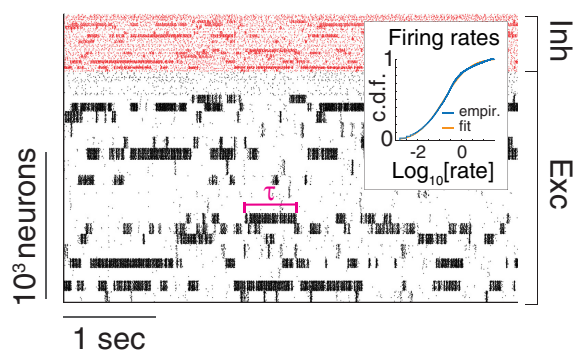

d State perturbation $\delta$ mean(E)

E-cell afferents

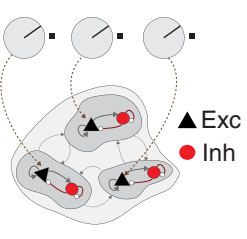
$\delta$ mean $(E)=10 \%$

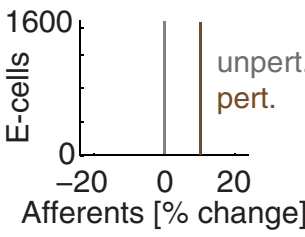

e State perturbation $\delta \operatorname{var}(E)$

C Inhibition stabilization

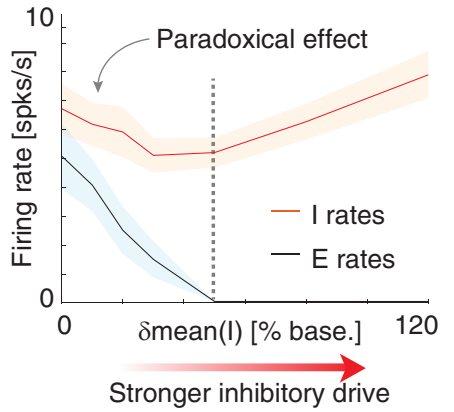

E-cell afferents $C V(E)=10 \%$
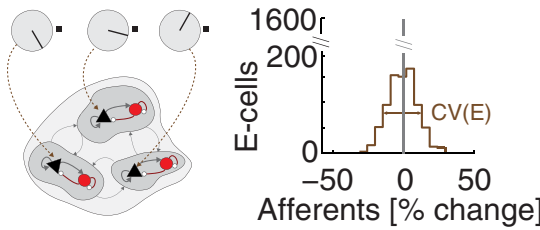

Figure 2. Biological plausible model of cortical circuit. $\boldsymbol{a}$, Left, Schematics of the network architecture. A recurrent network of $E$ (black triangles) and I (red circles) spiking neurons arranged in clusters is presented; sensory stimuli targeting subsets of $E$ clusters, in different cortical states implemented by perturbations. Inset, A membrane potential trace from representative $E$ neuron. Right, Synaptic couplings $J_{i j}$ for a representative clustered network, highlighting the block diagonal structure of potentiated intracluster synaptic weights for both $E$ and I clusters, and the background $E$ and I populations (bgr). Inset, Cluster size was heterogeneous. $\boldsymbol{b}$, Representative neural activity during ongoing periods. Tick marks represent spike times of $\mathrm{E}$ (black) or I (red) neurons. The network dynamics are metastable, with clusters transient activity for periods of duration $\tau$. Inset, The cumulative distributions of single-cell firing rates (in the representative network are log-normal; blue represents empirical data; orange represents log-normal fit). c, When increasing the inhibitory drive (afferent current to the I population, same as $\delta$ mean $(I)$ perturbation), both $\mathrm{E}$ and I firing rates decrease (right; black and red curve; mean \pm SEM across 10 simulated networks), highlighting the paradoxical effect, signature of the inhibition stabilized regimen (Tsodyks et al., 1997). Beyond $\delta$ mean $(I)=50 \%$, the E population shuts down and the I population rebounds (dashed vertical line). $\boldsymbol{d}$, Left, State-changing perturbation affecting the mean of the afferent currents to $E$ populations (knobs represent changes in afferent to three representative $E$ cells compared with the unperturbed state). Right, Histogram of afferent inputs to $E$ cells in the perturbed state (brown, all neurons receive a $10 \%$ increase in input) with respect to the unperturbed state (gray). $\boldsymbol{e}$, Left, State-changing perturbation affecting the variance of afferent currents to E populations. Right, In the perturbed state (brown), each E-cell's afferent input is constant and sampled from a normal distribution with mean equal to the unperturbed value (gray) and $10 \%$ CV.

gratings were presented at four directions $\left(0^{\circ}, 45^{\circ}, 90^{\circ}, 135^{\circ}\right)$ and one temporal frequency $(2 \mathrm{~Hz})$. Of the 54 sessions provided, only 7 sessions (6 male, 1 female) had enough trials per behavioral condition to perform our decoding analysis. Of these 7 sessions, 3 were recorded in WT male mice, 3 in transgenic male mice, and 1 in a transgenic female mouse. Neural activity from the visual cortical hierarchy was collected and, specifically: primary visual cortex (V1) in 5 of these 7 sessions, with a median value of 75 neurons per session; lateral visual area (LM): 6 sessions, 47 neurons; anterolateral visual area (AL): 5 sessions, 61 neurons; posteromedial visual area (PM): 6 sessions, 55; anteromedial visual area (AM): 7 sessions, 48 neurons. We matched the number and duration of trials across condition and orientation and combined trials from the drifting gratings repeat stimulus set, and drifting grating contrast stimulus set. 
a

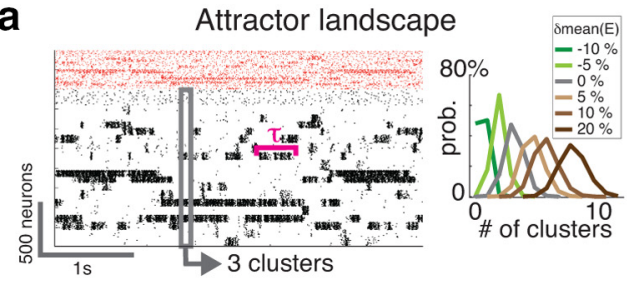

b Timescale and gain (simulations)

Perturbing mean afferents

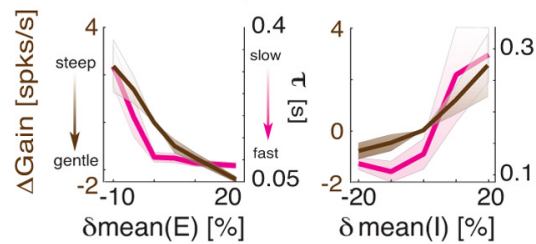

Perturbing afferent variance

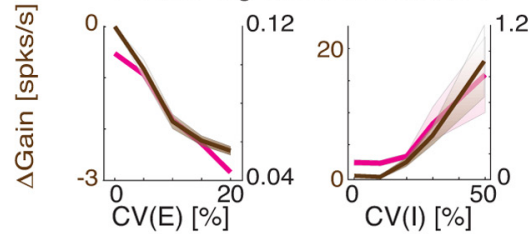

Perturbing synaptic couplings
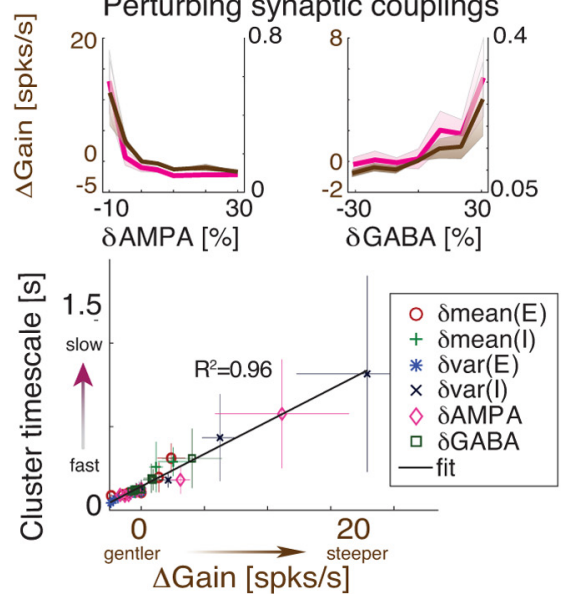

C Mean field theory

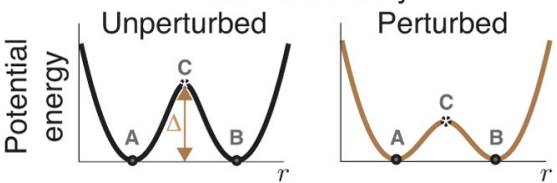

Attractors

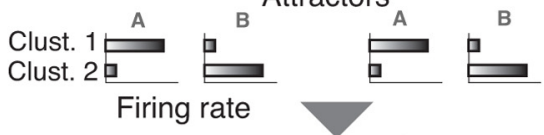

Potential energy: $E=\int d r[r-\Phi(r)]$

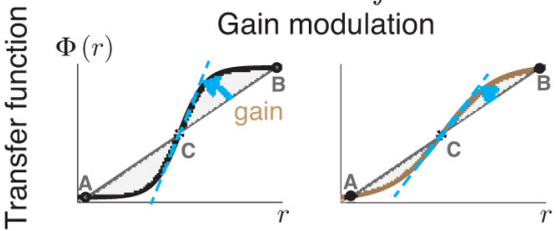

d Barrier heights and gain (mean field theory)
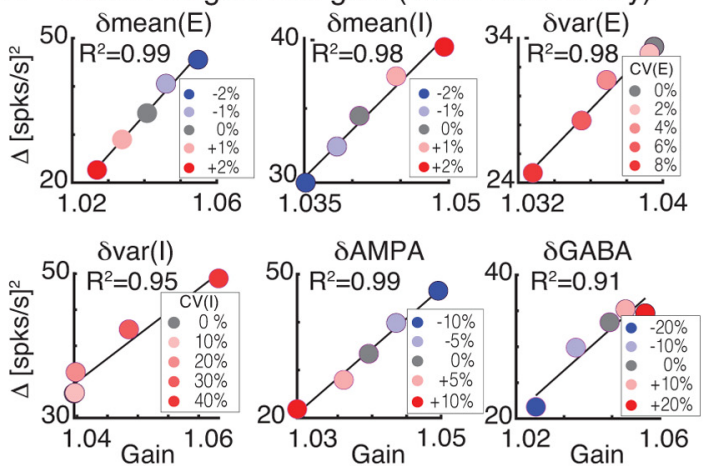

e Inferring transfer functions from spikes

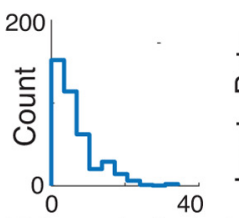

Firing rate [spks/s]
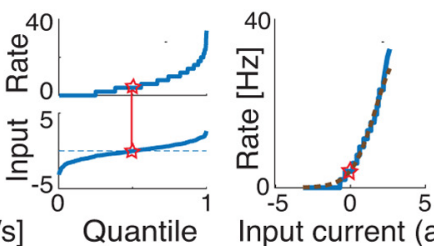

Input current (a.u.)

Figure 3. Linking gain modulation to changes in cluster timescale. $\boldsymbol{a}$, Clustered network activity during a representative unperturbed ongoing trial hops among different metastable attractors (gray box: attractor with 3 coactive clusters). Right, Perturbations strongly modulate the attractor landscape (color-coded curves: frequency of occurrence of network attractors with different number of coactive clusters, for different values of the representative $\delta$ mean(E) perturbation, mean occurrence across 5 sessions). $\boldsymbol{b}$, Perturbations induce consistent changes in the average cluster activation timescale $\tau$ (mean \pm SD across 10 simulated sessions) and in the single-neuron intrinsic gain ( $\Delta$ Gain = Gain(pert.) - Gain(unpert.), estimated as in $\boldsymbol{e}_{\boldsymbol{;}}$ bottom, color-coded markers represent different perturbations, linear regression, $\left.R^{2}=0.96\right)$. $c$, Schematic of the effect of perturbations on network dynamics in a two cluster network, captured by a double-well potential (top). Potential wells represent two attractors where either cluster is active (A and $B$; left, unperturbed), separated by a barrier with height $\Delta$. Mean field theory links perturbation effects on the barrier height (top right, lower barrier) to changes in the intrinsic neuronal gain (bottom right, lower gain). $\boldsymbol{d}$, Mean field theory predictions linking the height of the barrier $\Delta$ separating the attractors to the intrinsic cell gain, for all perturbations (linear regression of $\Delta$ vs gain). e, Inferring the single-cell intrinsic transfer function from spiking activity during ongoing periods. By recasting the distribution of spike counts (left) into quantiles (top center), one can match those values to corresponding quantiles of a Gaussian input current distribution (bottom center) to obtain the current-to-rate function (right). $\boldsymbol{d}$, A single-cell transfer function (bottom; blue represents empirical data; brown represents sigmoidal fit) can be estimated by matching a neuron's firing rate distribution during ongoing periods (top) to a Gaussian distribution of input currents (middle; quantile plots; red stars represent matched median values).

To do this, we combined trials with low-contrast gratings $(0.08,0.1,0.13$, $0.2)$ and trials with high-contrast gratings $(0.6,0.8,1)$ into separate trial types to perform the decoding analysis, and analyzed the interval $[-0.25$, $0.5 \mathrm{~s}$ ] aligned to stimulus onset.

For evoked activity, running trials were classified as those where the animal was running faster than $3 \mathrm{~cm} / \mathrm{s}$ for the first $0.5 \mathrm{~s}$ of stimulus presentation. During ongoing activity, behavioral periods were broken up into windows of $1 \mathrm{~s}$. Periods of running or rest were classified as such if $10 \mathrm{~s}$ had elapsed without a behavioral change. Blocks of ongoing activity were sorted and used based on the length of the behavior. Of the 54 sessions provided, 14 sessions had enough time per behavioral condition (minimum of $2 \mathrm{~min}$ ) to estimate single-cell transfer functions. Only neurons with a mean firing rate during ongoing activity $>5 \mathrm{~Hz}$ were included in the gain analysis (2119 of 4365 total neurons).

\section{Stimulus decoding}

For both the simulations and data, a multiclass decoder was trained to discriminate between four stimuli from single-trial population activity vectors in a given time bin (Jezzini et al., 2013). To create a time course of decoding accuracy, we used a sliding window of $100 \mathrm{~ms}(200 \mathrm{~ms})$ in the data (model), which was moved forward in $2 \mathrm{~ms}(20 \mathrm{~ms})$ intervals in the data (model). Trials were split into training and test datasets in a stratified fivefold cross-validated manner, ensuring equal proportions of trials per orientation in both datasets. In the model, a leave-2-out cross- 
Table 2. Parameters for the simplified two cluster network used for the meanfield theory analysis ${ }^{a}$

\begin{tabular}{lll}
\hline Parameter & Description & Value \\
\hline$j_{E E}$ & Mean E-to-E synaptic weights $\times \sqrt{N}$ & $0.8 \mathrm{mV}$ \\
$j_{E I}$ & Mean I-to-E synaptic weights $\times \sqrt{N}$ & $10.6 \mathrm{mV}$ \\
$j_{I E}$ & Mean E-to-I synaptic weights $\times \sqrt{N}$ & $2.5 \mathrm{mV}$ \\
$j_{I I}$ & Mean I-to-I synaptic weights $\times \sqrt{N}$ & $9.7 \mathrm{mV}$ \\
$j_{E 0}$ & Mean E-to-E synaptic weights $\times \sqrt{N}$ & $14.5 \mathrm{mV}$ \\
$j_{I 0}$ & Mean I-to-I synaptic weights $\times \sqrt{N}$ & $12.9 \mathrm{mV}$ \\
$J_{E E}^{+}$ & Potentiated intracluster E-to-E weight factor & 11.2 \\
$r^{e x t}$ & Average baseline afferent rate to E and I neurons & $7 \mathrm{spikes} / \mathrm{s}$ \\
$V_{E}^{\text {thr }}$ & E-neuron threshold potential & $4.6 \mathrm{mV}$ \\
$V_{I}^{\text {thr }}$ & I-neuron threshold potential & $8.7 \mathrm{mV}$ \\
$\tau_{S}$ & E- and I-neuron synaptic time constant & $4 \mathrm{~ms}$ \\
$n_{b g r}$ & Fraction of background E neurons & $65 \%$ \\
\hline
\end{tabular}

${ }^{a}$ The remaining parameters are in Table 1 .

validation was performed. To calculate the significance of the decoding accuracy, an iterative shuffle procedure was performed on each fold of the cross-validation. On each shuffle, the training labels were shuffled and the classifer accuracy was predicted on the unshuffled test dataset. This shuffle was performed 100 times to create a shuffle distribution to rank the actual decoding accuracy from the unshuffled decoder against and to determine when the mean decoding accuracy had increased above chance. This time point is what we referred to as the latency of stimulus decoding. To account for the speed of stimulus decoding (the slope of the decoding curve), we defined the $\Delta$-Latency between running and rest as the average time between the two averaged decoding curves from $40 \%$ up to $80 \%$ of the max decoding value at rest.

\section{Firing rate distribution match}

To control for increases of firing rate because of locomotion (see Fig. $7 b$ ), we matched the distributions of population counts across the trials used for decoding in both behavioral conditions. This procedure was done independently for each sliding window of time along the decoding time course. Within each window, the spikes from all neurons were summed to get a population spike count per trial. A log-normal distribution was fit to the population counts across trials for rest and running before the distribution match (see Fig. $8 a$, left). We sorted the distributions for rest and running in descending order, randomly removing spikes from trials in the running distribution to match the corresponding trials in the rest distribution (see Fig. $8 a$, right). By doing this, we only removed the number of spikes necessary to match the running distribution to rest distribution. For example, in trials where the rest distribution had a larger population count, no spikes were removed from either distribution. Given that we performed this procedure at the population level rather than per neuron, we checked the change in peristimulus time histogram (PSTH) between running and rest conditions before and after distribution matching (see Fig. 8b).

Single-cell gain

To infer the single-cell transfer function in simulations and data, we followed the method originally described by Recanatesi et al. (2020) (for a trial-averaged version, see also Lim et al., 2015; Pereira and Brunel, 2018). We estimated the transfer function on ongoing periods when no sensory stimulus was present. Briefly, the transfer function of a neuron was calculated by mapping the quantiles of a standard Gaussian distribution of input currents to the quantiles of the empirical firing rate distribution during ongoing periods (see Fig. 3e). We then fit this transfer function with a sigmoidal function. The max firing rate of the neuron in the sigmoidal fit was bounded to be no larger than 1.5 times that of the empirical max firing rate, to ensure realistic fits. We defined the gain as the slope at the inflection point of the sigmoid.

Single-cell response and selectivity

We estimated the proportion of neurons that were significantly excited or inhibited by cortical state perturbations in the model (see Fig. 6) or locomotion in the data (see Fig. 7) during periods of ongoing activity, in the absence of sensory stimuli. In the model, we simulated 40 trials per network, for 10 networks per each value of the perturbation. Only in this perturbation-response condition in the absence of sensory stimuli, the perturbation was modeled as a double exponential with rise and decay times $[0.2,1]$ (see Fig. $3 a$ ), with $0.5 \mathrm{~s}$ of unperturbed activity preceding perturbation onset. In the data, we binned the spike counts in $500 \mathrm{~ms}$ windows for each neuron after matching sample size between rest and running conditions, and significant difference between the ongoing and perturbed epochs was assessed with a rank-sum test.

We estimated single neuron selectivity to sensory stimuli in each condition from the average firing rate responses $r_{i}^{a}(t)$ of the $i$-th neuron to stimulus $a$ in trial $t$. For each pair of stimuli, selectivity was estimated as follows:

$$
d^{\prime}(a, b)=\frac{\operatorname{mean}\left[r(t)^{a}\right]-\operatorname{mean}\left[r(t)^{b}\right]}{\sqrt{\frac{1}{2}\left(\operatorname{var}\left[r(t)^{a}\right]+\operatorname{var}\left[r(t)^{b}\right]\right)}}
$$

where mean and var are estimated across trials. The $d^{\prime}$ was then averaged across stimulus pairs.

\section{Results}

To elucidate the effect of state changes on cortical dynamics, we modeled the local circuit as a network of recurrently connected $\mathrm{E}$ and I spiking neurons. Both E and I populations were arranged in clusters (Amit and Brunel, 1997; Litwin-Kumar and Doiron, 2012; Mazzucato et al., 2015, 2019; Schaub et al., 2015), where synaptic couplings between neurons in the same cluster were potentiated compared with neurons in different clusters, reflecting the empirical observation of cortical assemblies of functionally correlated neurons (Fig. 2a) (Song et al., 2005; Perin et al., 2011; Kiani et al., 2015; W. C. Lee et al., 2016). In the absence of external stimulation (ongoing activity), this E-I clustered network generates rich temporal dynamics characterized by metastable activity operating in inhibition stabilized regimen, where E and I postsynaptic currents track each other, achieving tight balance (Fig. 2c). A heterogeneous distribution of cluster sizes leads to a log-normal distribution of firing rates (Fig. $2 b$ ). Network activity was characterized by the emergence of the slow timescale of cluster transient activation, with average activation lifetime of $\tau=106 \pm 35 \mathrm{~ms}$ (hereby referred to as "cluster timescale"; Fig. 2b), much larger than single-neuron time constant $(20 \mathrm{~ms})$ (LitwinKumar and Doiron, 2012; Mazzucato et al., 2015).

To investigate how changes in cortical state may affect the network dynamics and information processing capabilities, we examined a vast range of state-changing perturbations (Fig. $2 d, e$; Table 3). State changes were implemented as perturbations of the afferent currents to cell type-specific populations, or as perturbations to the synaptic couplings. The first type of state perturbations $\delta$ mean(E) affected the mean of the afferent currents to $\mathrm{E}$ populations (Fig. $2 d$ ). For example, a perturbation $\delta$ mean $(\mathrm{E})=$ $10 \%$ implemented an increase of all input currents to E neurons by $10 \%$ above their unperturbed levels. The perturbation $\delta$ mean (I) affected the mean of the afferent currents to I populations in an analogous way. The second type of state perturbations $\delta$ var (E) affected the across-neuron variance of afferents to E populations. Namely, in this perturbed state, the afferent current to each neuron in that population was sampled from a normal distribution with zero mean and fixed variance (Fig. $2 e$, measured by the coefficient of variability $\mathrm{CV}(\mathrm{E})=\operatorname{var}(\mathrm{E}) /$ mean $(\mathrm{E})$ with respect to the unperturbed afferents). This perturbation thus 
Table 3. Classification of state-changing perturbations

\begin{tabular}{llllll}
\hline & Latency & Activity & Response & $\tau$ & Gain \\
\hline$\delta$ mean $(E) \nearrow$ & $\searrow$ & $\mathrm{E}[\nearrow], \mathrm{I}[\nearrow]$ & $\mathrm{E}[\nearrow], \mathrm{I}[\nearrow]$ & $\searrow$ & $\searrow$ \\
$\delta$ mean $(I)$ & $\nearrow$ & $\mathrm{E}[\searrow], \mathrm{I}[\searrow]$ & $\mathrm{E}[\searrow], \mathrm{I}[\mathrm{mixed}]$ & $\nearrow$ & $\nearrow$ \\
$\delta \operatorname{var}(E) \nearrow$ & $\searrow$ & $\mathrm{E}[\nearrow], \mathrm{I} / \nearrow]$ & $\mathrm{E}[$ mixed], I[mixed] & $\searrow$ & $\searrow$ \\
$\delta \operatorname{var}(I) \nearrow$ & $\nearrow$ & $\mathrm{E}[\searrow], \mathrm{I}[=]$ & $\mathrm{E}[\searrow], \mathrm{I}[\searrow]$ & $\nearrow$ & $\nearrow$ \\
$\delta A M P A$ & $\searrow$ & $\mathrm{E}[\nearrow], \mathrm{I}[\nearrow]$ & $\mathrm{E}[\nearrow], \mathrm{I}[\nearrow]$ & $\searrow$ & $\searrow$ \\
$\delta G A B A \nearrow$ & $\nearrow$ & $\mathrm{E}[\searrow], \mathrm{I}[\searrow]$ & $\mathrm{E}[\searrow], \mathrm{I}[$ mixed] & $\nearrow$ & $\nearrow$ \\
Locomotion & $\searrow$ & $\mathrm{E}[\nearrow], \mathrm{I}[\nearrow]$ & $\mathrm{E}[$ mixed], I[mixed] & $\searrow$ & $\searrow$ \\
\hline
\end{tabular}

Effect of neural activity of an increasing ( $/$ ) state-changing perturbation: latency of stimulus decoding (Latency, Fig. 2e); average firing rate modulation (Activity) and response to perturbations (Response, proportion of cells with significant responses) of $E$ and I cells in the absence of stimuli (Fig. 3); cluster activation timescale $(\tau$, Fig. $4 b)$; single-cell intrinsic gain modulation at rest (Gain, Fig. 5e). $\nearrow$, increase; decrease; $=$, no change. Mixed responses refer to similar proportions of $E$ and I cells. The effect of locomotion is consistent with a perturbation increasing $\delta \operatorname{var}(\mathrm{E})$.

introduced a spatial variance across neurons in the cell type-specific afferent currents yet left the mean afferent current into the population unchanged. The state perturbation $\delta$ var(I) affected the variance of the afferent currents to I populations analogously. In the third type of state perturbations $\delta$ AMPA or $\delta \mathrm{GABA}$, we changed the average GABAergic or glutamatergic (AMPA) recurrent synaptic weights compared with their unperturbed values. We chose the range of state perturbations such that the network still retained nontrivial metastable dynamics within the whole range. We will refer to these state changes of the network as simply perturbations, and should not be confused with the presentation of the stimulus. We first established the effects of perturbations on ongoing network dynamics and used those insight to explain their effects on stimulus-evoked activity.

\section{State-dependent regulation of the network emergent timescale}

A crucial feature of neural activity in clustered networks is metastable attractor dynamics, characterized by the emergence of a long timescale of cluster activation whereby network itinerant activity explores the large attractor landscape (Fig. 2b). We first examined whether perturbations modulated the network's metastable dynamics and introduced a protocol where perturbations occurred in the absence of sensory stimuli ("ongoing activity").

We found that perturbations strongly modulated the attractor landscape, changing the repertoire of attractors the network activity visited during its itinerant dynamics (Fig. 3a). Changes in attractor landscape were perturbation-specific. Perturbations increasing $\delta$ mean(E) $(\delta$ mean(I)) induced a consistent shift in the repertoire of attractors: larger perturbations led to larger (smaller) numbers of coactive clusters. Surprisingly, perturbations that increased $\delta \operatorname{var}(\mathrm{E})$ $(\delta \operatorname{var}(\mathrm{I}))$ led to network configurations with larger (smaller) sets of coactivated clusters. This effect occurred despite that such perturbations did not change the mean afferent input to the network. Perturbations affecting $\delta$ AMPA and $\delta$ GABA had similar effects to $\delta$ mean $(\mathrm{E})$ and $\delta$ mean $(\mathrm{I})$, respectively.

We then examined whether perturbations affected the cluster activation timescale. We found that perturbations differentially modulated the average cluster activation timescale $\tau$ during ongoing periods, in the absence of stimuli (Fig. $3 b$ ). In particular, increasing $\delta$ mean $(\mathrm{E}), \delta \operatorname{var}(\mathrm{E})$, or $\delta$ AMPA led to a proportional acceleration of the network metastable activity and shorter $\tau$, whereas increasing $\delta$ mean(I), $\delta \operatorname{var}(\mathrm{I})$, or $\delta \mathrm{GABA}$ induced the opposite effect with longer $\tau$. Changes in $\tau$ were congruent with changes in the duration of intervals between consecutive activations of the same cluster (cluster interactivation intervals).

\section{Changes in cluster timescale are controlled by gain modulation}

What is the computational mechanism mediating the changes in cluster timescale, induced by the perturbations? We investigated this question using mean field theory, where network attractors, defined by sets of coactivated clusters, are represented as potential wells in an attractor landscape (Mascaro and Amit, 1999; Litwin-Kumar and Doiron, 2012; Mattia and Sanchez-Vives, 2012; Mazzucato et al., 2015, 2019). Let us illustrate this in a simplified network with two clusters (Fig. $3 c$ ). Here, the attractor landscape consists of two potential wells, each well corresponding to a configuration where one cluster is active and the other is inactive. When the network activity dwells in the attractor represented by the left potential well, it may escape to the right potential well because of internally generated variability. This process will occur with a probability determined by the height $\Delta$ of the barrier separating the two wells: the higher the barrier, the less likely the transition (Hänggi et al., 1990; Litwin-Kumar and Doiron, 2012; Mattia and Sanchez-Vives, 2012; Mazzucato et al., 2019).

Mean field theory thus established a relationship between the cluster timescale and the height of the barrier separating the two attractors. We found that perturbations differentially control the height of the barrier $\Delta$ separating the two attractors (Fig. $3 d$ ), explaining the changes in cluster timescale observed in the simulations (Fig. 3b).

Since reconstruction of the attractor landscape requires knowledge of the network's structural connectivity, the direct test of the mean field relation between changes in attractor landscape and timescale modulations is challenging. We thus aimed at obtaining an alternative formulation of the underlying neural mechanism only involving quantities directly accessible to experimental observation. Using mean field theory, one can show that the double potential well representing the two attractors can be directly mapped to the effective transfer function of a neural population (Mascaro and Amit, 1999; Mattia et al., 2013; Mazzucato et al., 2019). One can thus establish a direct relationship between changes in the slope (hereby referred to as "gain") of the intrinsic transfer function estimated during ongoing periods and changes in the barrier height $\Delta$ separating metastable attractors (Fig. $3 c, d$; see Materials and Methods). In turn, this implies a direct relationship between gain modulation, induced by the perturbations, and changes in cluster activation timescale. In particular, perturbations inducing steeper gain will increase well depths and barrier heights, and thus increase the cluster timescale, and vice versa. Using mean field theory, we demonstrated a complete classification of the differential effect of all perturbations on barrier heights and gain (Fig. $3 d$ ).

We then proceeded to verify these theoretical predictions, obtained in a simplified two cluster network, in the high dimensional case of large networks with several clusters using simulations. While barrier heights and the network's attractor landscape can be exactly calculated in the simplified two cluster network, this task is infeasible in large networks with a large number of clusters where the number of attractors is exponential in the number of clusters. On the other hand, changes in barrier heights $\Delta$ are equivalent to changes in gain, and the latter can be easily estimated from spiking activity (Fig. $3 b-d$ ). We thus tested whether the relation between gain and timescale held in the high-dimensional case of a network with many clusters. We estimated single-cell transfer functions from their spiking activity during ongoing periods, in the absence of sensory stimuli but in the presence of different perturbations (Fig. 3e) (Lim et al., 2015; 

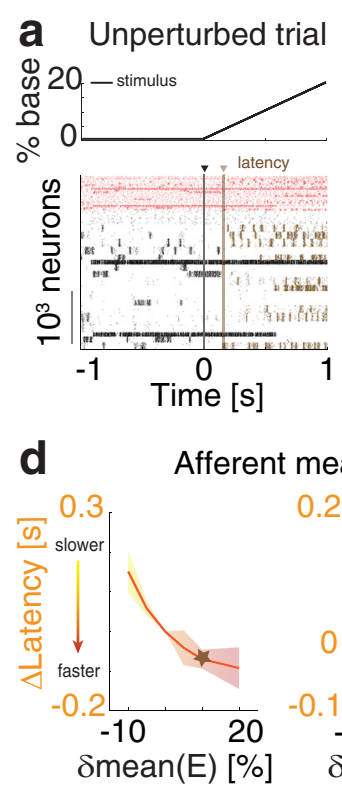
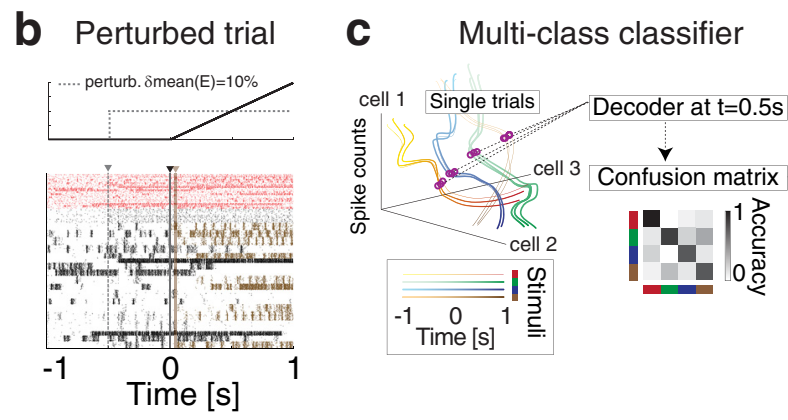

Stimulus-decoding

mean(hits)

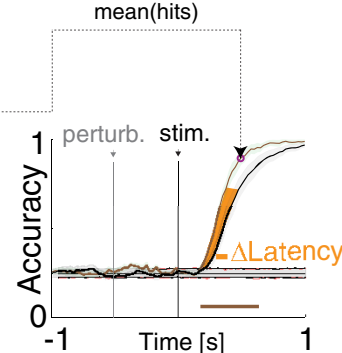

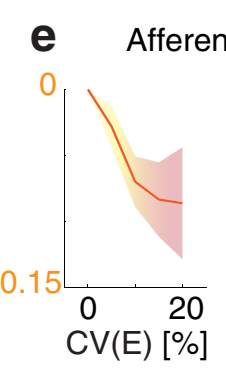
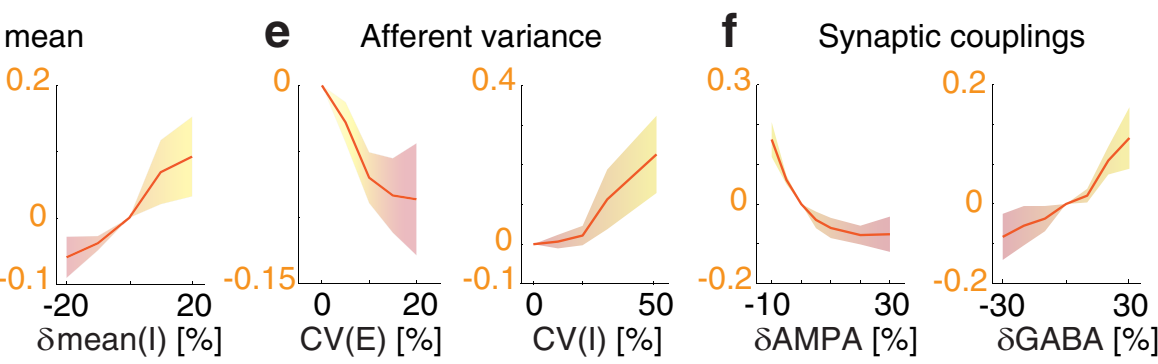

Figure 4. Perturbations control stimulus processing speed in the clustered network. $\boldsymbol{a}, \boldsymbol{b}$, Representative trials in the unperturbed $(\boldsymbol{a})$ and perturbed (b) conditions; the representative perturbation is an increase in the spatial variance $\delta \operatorname{var}(\mathrm{E})$ across $\mathrm{E}$ neurons. After a ramping stimulus is presented at $t=0$ (black vertical line on raster plot; top, stimulus time course), stimulus-selective $\mathrm{E}$ clusters (brown tick marks represent their spiking activity) are activated at a certain latency (brown vertical line). In the perturbed condition (b), a perturbation is turned on before stimulus onset (gray dashed vertical line). The activation latency of stimulus-selective clusters is shorter in the perturbed compared with the unperturbed condition. $c$, Left, Schematic of stimulus-decoding analysis. A multiclass classifier is trained to discriminate between the four stimuli from single-trial population activity vectors in a given time bin (curves represent the time course of population activity in single trials, color-coded for four stimuli; purple circle highlights a given time bin along the trajectories), yielding a cross-validated confusion matrix for the decoding accuracy at that bin (middle). Right, Average time course of the stimulus-decoding accuracy in the unperturbed (black) and perturbed (brown) conditions (horizontal brown: significant difference between conditions, $p<0.05$ with multiple bin correction). $\boldsymbol{d}$-f, Difference in stimulus decoding latency in the perturbed minus the unperturbed conditions (average difference between decoding accuracy time courses in the $[40 \%, 80 \%]$ range, yellow interval of $c$; mean \pm SD across 10 networks) for the six state-changing perturbations (for details, see Materials and Methods and main text). Brown star represents the perturbation in $\boldsymbol{b}, \boldsymbol{c}$.

Recanatesi et al., 2020). We found that network perturbations strongly modulated single-cell gain in the absence of stimuli, verifying mean field theory predictions in all cases (Fig. 3e). In particular, we confirmed the direct relationship between gain modulation and cluster timescale modulation: perturbations that decreased (increased) the gain also decreased (increased) cluster timescale (Fig. $3 b ; R^{2}=0.96$ ). For all perturbations, gain modulations explained the observed changes in cluster timescale.

\section{Controlling information processing speed with perturbations}

We found that changes in cortical state during ongoing activity, driven by external perturbations, control the circuit's dynamical timescale. The neural mechanism mediating the effects of external perturbations is gain modulation, which controls the timescale of the network switching dynamics. How do such state changes affect the network information processing speed?

To investigate the effect of state perturbations on the network's information processing, we compared stimulus-evoked activity by presenting stimuli in an unperturbed and a perturbed condition. In unperturbed trials (Fig. $4 a$ ), we presented one of four sensory stimuli, modeled as depolarizing currents targeting a subset of stimulus-selective $\mathrm{E}$ neurons with linearly ramping time course. Stimulus selectivities were mixed and random, all clusters having equal probability of being stimulus-selective. In perturbed trials (Fig. 4b), in addition to the same sensory stimuli, we included a state perturbation, which was turned on before the stimulus and was active until the end of stimulus presentation. We investigated and classified the effect of several state changes implemented by perturbations affecting either the mean or variance of cell type-specific afferents to $\mathrm{E}$ or I populations, and the synaptic couplings. State perturbations were identical in all trials of the perturbed condition for each type; namely, they did not convey any information about the stimuli.

We assessed how much information about the stimuli was encoded in the population spike trains at each moment using a multiclass classifier (with four class labels corresponding to the four presented stimuli; Fig. 4c). In the unperturbed condition, the time course of the cross-validated decoding accuracy, averaged across stimuli, was significantly above chance after $0.21 \pm 0.02 \mathrm{~s}$ (mean \pm SEM across 10 simulated networks; Fig. $4 c$, black curve) and reached perfect accuracy after $1 \mathrm{~s}$. In the perturbed condition, stimulus identity was decoded at chance level in the period after the onset of the state perturbation but before stimulus presentation (Fig. $4 c$ ), consistent with the fact that the state perturbation did not convey information about the stimuli. We found that state perturbations significantly modulated the network information processing speed. We quantified this modulation as the average latency to reach a decoding accuracy between $40 \%$ and $80 \%$ (Fig. $4 c$, yellow area), and found that state perturbations differentially affected processing speed.

State perturbations had opposite effects depending on which cell type-specific populations they targeted. Increasing $\delta$ mean (E) monotonically improved network performance (Fig. $4 d$, left): in particular, positive perturbations induced an anticipation of stimulus-coding (shorter latency), whereas negative ones led to longer latency and slower coding. The opposite effect was achieved when increasing $\delta$ mean(I), which slowed down processing speed (Fig. $4 d$, right). State perturbations that changed the spatial variance of the afferent currents had counterintuitive 
a Selective-cluster onset
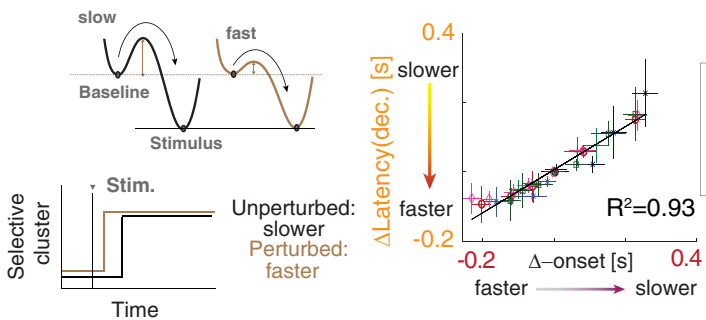

b Cluster timescale C Gain modulation

d Cluster timescale and processing speed

Peturbing mean afferents

Peturbing afferent variance

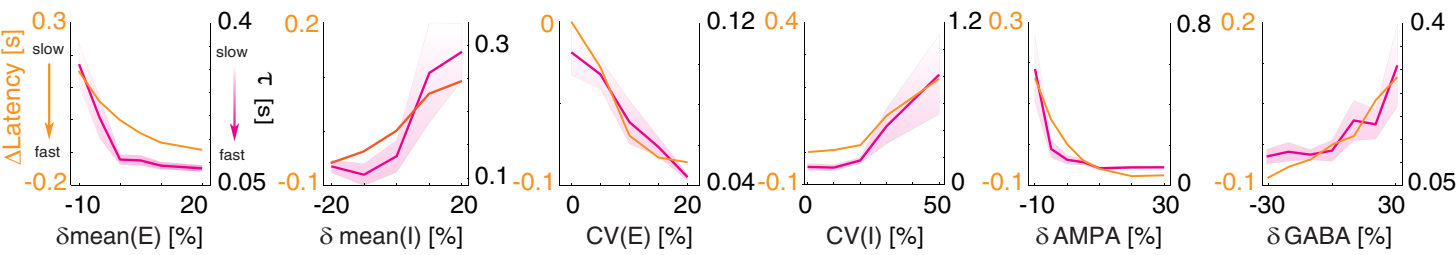

e Gain modulation and processing speed
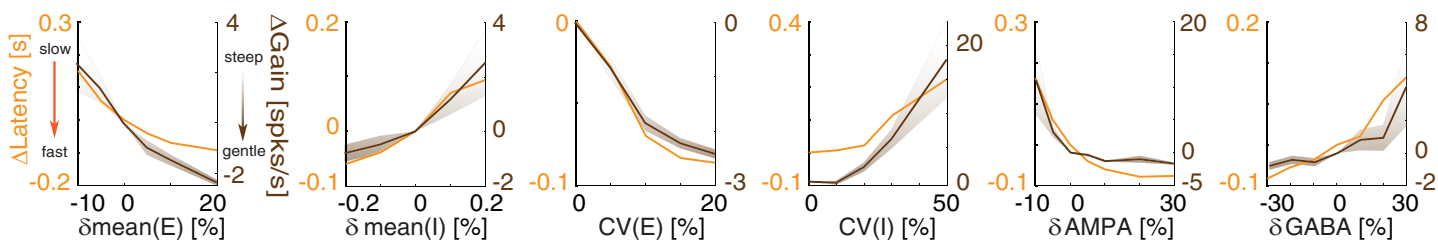

Figure 5. Linking gain modulation to changes in processing speed. $\boldsymbol{a}$, Left, Schematic of the effect of an accelerating perturbation on stimulus encoding during evoked activity (same notations as in Fig. 3c). A shrinking barrier from nonselective to selective attractors drives a faster activation of stimulus-selective cluster after stimulus presentation. Right, Changes in stimulus processing speed ( $y$ axis: latency of stimulus decoding from Fig. $4 d-f$ ) are predicted by changes in activation latency of stimulus-selective clusters ( $x$ axis: mean \pm SD across 10 simulated sessions; linear regression, $\left.R^{2}=0.93\right)(\boldsymbol{b}, \boldsymbol{d})$ by changes in cluster timescale (same values as in Fig. $\left.3 b ; R^{2}=0.93\right)$; and $(\boldsymbol{c}, \boldsymbol{e})$ by changes in single-cell intrinsic gain (same values as in Fig. $\left.3 b ; R^{2}=0.71\right)$.

effects (Fig. 4e). We measured the strength of these perturbations via their coefficient of variability $C V(\alpha)=\sigma_{\alpha} / \mu_{\alpha}$, for $\alpha=E, I$, where $\sigma$ and $\mu$ are the SD and mean of the across-neuron distribution of afferent currents. Perturbations $\delta$ var(E) that increased $C V(E)$ led to faster processing speed. The opposite effect was achieved with perturbations $\delta \operatorname{var}(\mathrm{I})$ inducing a spatial variance across afferents to I neurons, which slowed down stimulus processing speed (Fig. 4e). Perturbations $\delta$ AMPA which increased the glutamatergic synaptic weights improved performance proportionally to the perturbation. The opposite effect was achieved by perturbations $\delta$ GABA that increased the GABAergic synaptic weights, which monotonically decreased network processing speed (Fig. 4f). We thus concluded that afferent current perturbations differentially modulated the speed at which network activity encoded information about incoming sensory inputs. Such modulations exhibited a rich dynamical repertoire (Table 3 ).

\section{Gain modulation regulates the network information processing speed}

Our mean field framework demonstrates a direct relationship between the effects of perturbations on the network information processing speed and its effects on the cluster timescale (Fig. 3). In our simplified network with two clusters, stimulus presentation induces an asymmetry in the well depths, where the attractor $\mathrm{B}$ corresponding to the activated stimulus-selective cluster has a deeper well, compared with the attractor A where the stimulus-selective cluster is inactive. Upon stimulus presentation, the network ongoing state will be biased to transition toward the stimulus-selective attractor $\mathrm{B}$ with a transition rate determined by the barrier height separating $\mathrm{A}$ to $\mathrm{B}$. Because external perturbations regulate the height of such barrier via gain modulation, they control in turn the latency of activation of the stimulus-selective cluster. We thus aimed at testing the prediction of our theory: that the perturbations modulate stimulus coding latency in the same way as they modulate cluster timescales during ongoing periods; and, as a consequence, that these changes in stimulus coding latency can be predicted by intrinsic gain modulation. Specifically, our theory predicts that perturbation driving a decrease (increase) in intrinsic gain during ongoing periods will induce a faster (slower) encoding of the stimulus.

We thus proceeded to test the relationship between perturbations effects on cluster timescales, gain modulation, and information processing speed. In the representative trial where the same stimulus was presented in the absence (Fig. $4 a$ ) or in the presence (Fig. $4 b$ ) of the perturbation $\delta$ mean $(\mathrm{E})=10 \%$, we found that stimulus-selective clusters (highlighted in brown) had a faster activation latency in response to the stimulus in the perturbed condition compared with the unperturbed one. A systematic analysis confirmed this mechanism showing that, for all perturbations, the activation latency of stimulus-selective clusters was a strong predictor of the change in decoding latency (Fig. $5 a$, right; $\left.R^{2}=0.93\right)$. Moreover, we found that the perturbation-induced changes of the cluster timescale $\tau$ during ongoing periods predicted the effect of the perturbation on stimulus processing latency during evoked periods (Fig. $5 b, d$ ). Specifically, perturbations inducing faster $\tau$ during ongoing periods in turn accelerated stimulus coding, and vice versa for perturbations inducing slower $\tau$.

We then tested whether perturbation-induced gain modulations during ongoing periods explained the changes in stimulus 
a Responses to perturbations

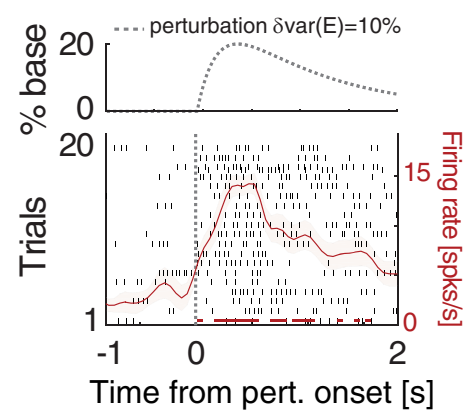

b
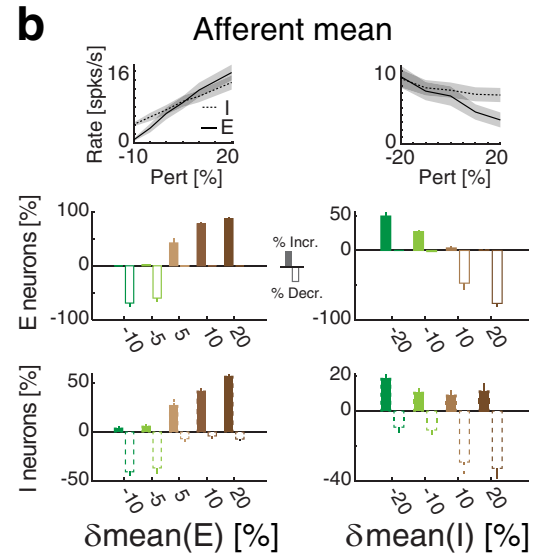
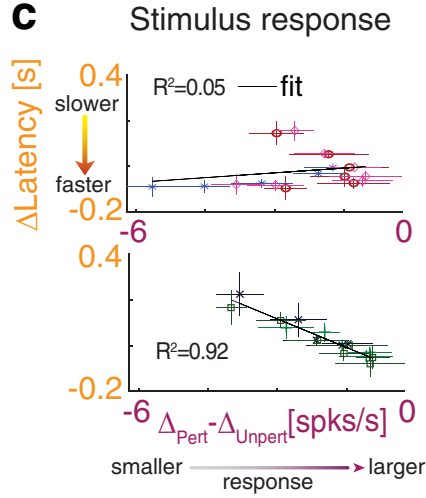

Afferent variance
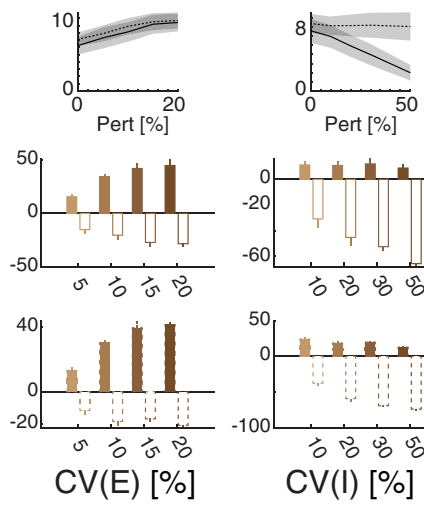

d Stimulus selectivity

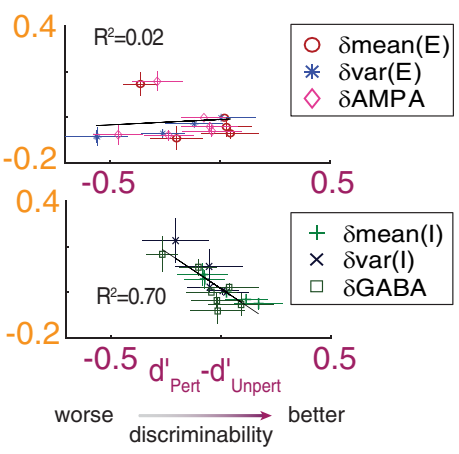

Synaptic couplings
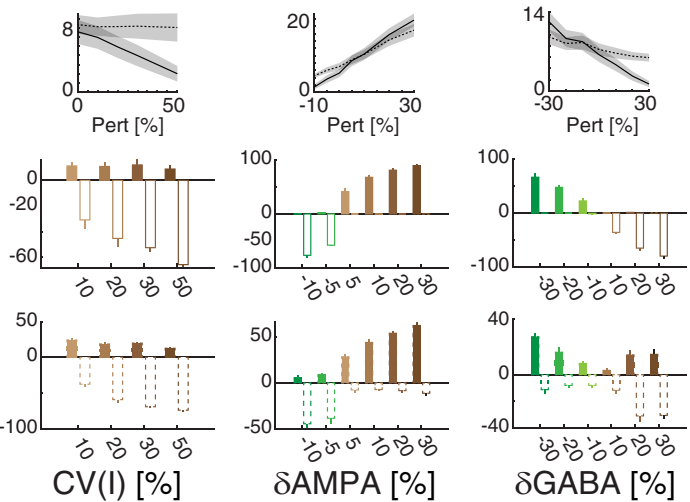

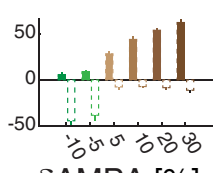

SAMPA [\%]

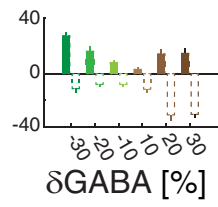

Figure 6. Single-cell responses to perturbations. $\boldsymbol{a}$, Representative single-cell response to the perturbation $\delta$ var $(E)=10 \%$ in the absence of stimuli (top: dashed line, time course of perturbation, occurring at $t=0$; bottom: dashed line, perturbation onset; red curve, response PSTH, mean \pm SD across 20 trials; horizontal red bar, significant response, $t$ test, $p<0.05$ with multiple bin correction). $\boldsymbol{b}$, Top, Average firing rate change across $\mathrm{E}$ (full) and I (dashed) populations in response to each state-changing perturbation (mean \pm SD across 10 simulated networks). Histograms represent average fractions of $\mathrm{E}$ (top row) and I (bottom row) neurons whose firing rate significantly increases (positive bars) or decreases (negative bars) in response to the perturbations (single-cell significant response was based on a $t$ test of the baseline vs perturbation-evoked activity, $p<0.05)$. $\boldsymbol{c}, \boldsymbol{d}$, Single-cell changes in firing rate response to stimuli $(\Delta=$ peak response-baseline in each perturbed or unperturbed condition) as well as changes in stimulus selectivity [ $d^{\prime}$ (perturbed trials) - $d^{\prime}$ (unperturbed trials)] because the perturbations are overall uncorrelated to changes in stimulus-decoding latency (mean \pm SEM across 5 networks).

processing speed during evoked periods, and found that the theoretical prediction was borne out in the simulations (Fig. $5 c, e$ ). Let us summarize the conclusion of our theoretical analyses. Motivated by mean field theory linking gain modulation to changes in transition rates between attractors, we found that gain modulation controls the cluster timescale during ongoing periods, and, in turn, regulates the onset latency of stimulus-selective clusters on stimulus presentation. Changes in onset latency of stimulus-selective clusters explained changes in stimulus-coding latency. We thus linked gain modulation to changes in stimulus processing speed (Fig. 5; Table 3).

\section{Physiologic responses to perturbations}

Our results show that cortical processing speed can be accelerated or slowed down via external perturbations. We found that different types of perturbations may induce similar effects on processing speed: a dynamical acceleration may be obtained by either increasing the mean or the variance of the external input to E neurons, or either decreasing the mean or the variance of external inputs to I neurons. A dynamical deceleration may be obtained by the opposite perturbations. In order to devise an experimental test of our theory to dissect the specific effects of each type of perturbation, we then examined the single-cell responses to perturbations. By combining single-cell responses with dynamical effects, we will be able to isolate the effects of each perturbation.
We characterized single-cell responses to perturbations during ongoing periods, in the absence of sensory stimuli (Fig. 6). For this perturbation-only condition, we introduced perturbation time course modeled as a double exponential, to estimate single-cell responses to a physiologically plausible perturbation profile in the absence of sensory stimuli. We found that perturbations differentially affected neuronal responses in a cell typespecific way. Perturbations changed the average population firing rates and led to complex patterns of response across $\mathrm{E}$ and I populations (Fig. 6). Specifically, perturbations increasing $\delta$ mean(E) induced higher firing rates and induced proportionally excited responses in both $\mathrm{E}$ and I populations. On the other hand, perturbations that increased $\delta$ mean(I) led to a decrease in both $\mathrm{E}$ and I average firing rates. This paradoxical effect (Tsodyks et al., 1997) revealed that the network operates in the inhibition stabilized regimen (Fig. 2c). When increasing the inhibitory current beyond $\delta$ mean $(\mathrm{I})=50 \%$, the network reached a reversal point where the $\mathrm{E}$ population activity became silent and the I population rebounded, starting to increase their firing rates again (Fig. $2 c$ ).

Perturbations increasing the variance $\delta \operatorname{var}(\mathrm{E})$ and $\delta \operatorname{var}(\mathrm{I})$ led to surprising effects (Fig. 6). Increasing $\delta \operatorname{var}(\mathrm{E})$ induced higher firing rates in both $\mathrm{E}$ and I populations, despite leaving the mean afferents unchanged; moreover, it led to mixed responses at the single-cell level, with a prevalence of excited responses in both $\mathrm{E}$ and I populations. We will see below that this set of responses is 

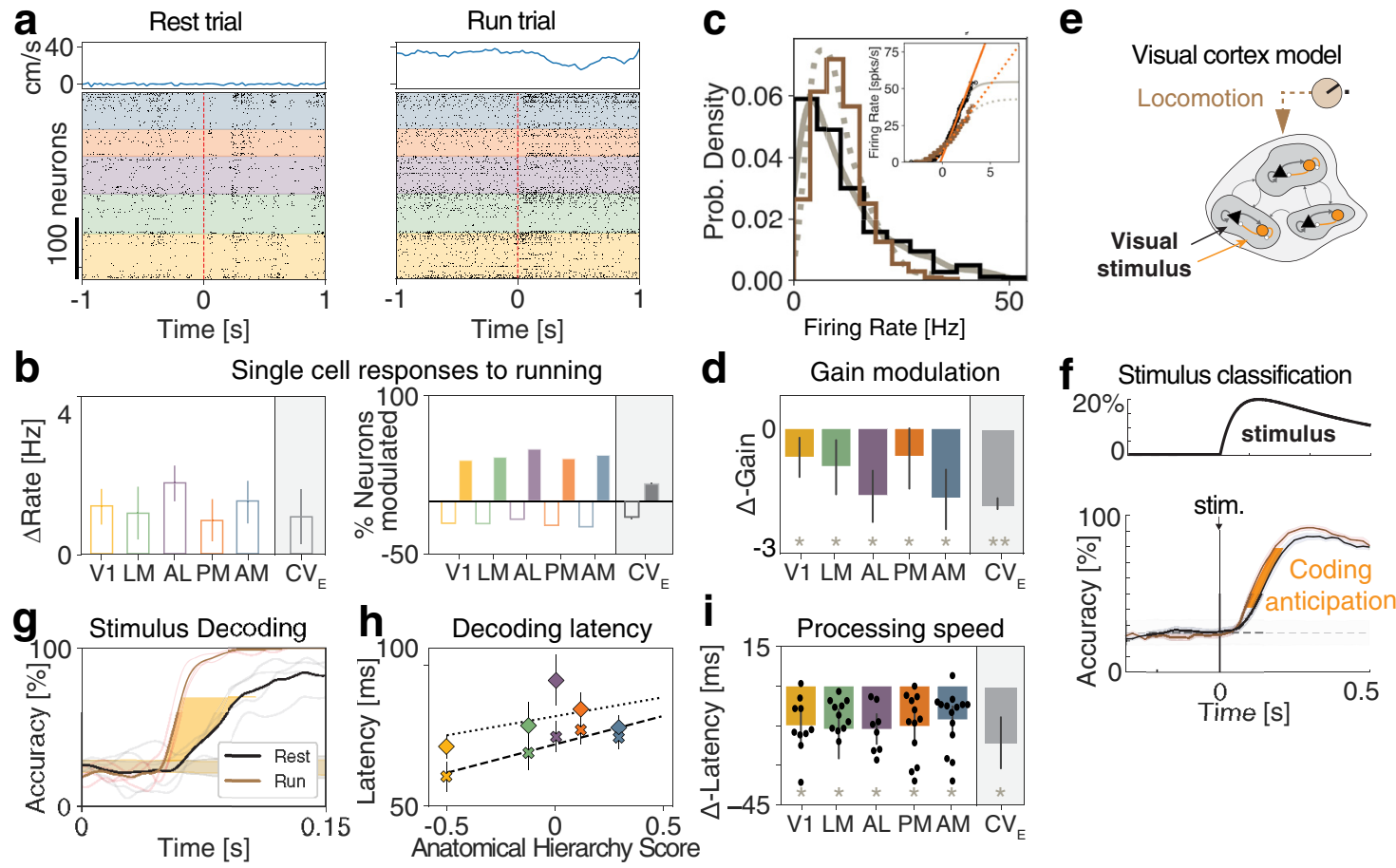

Figure 7. Locomotion effects on visual processing are mediated by gain modulation. $\boldsymbol{a}$, Representative raster plots from five cortical visual areas (color-coded) with population spiking activity during passive presentation of drifting gratings (dashed red line indicates stimulus onset) during periods of running (right, running speed in top panels) and rest (left). $\boldsymbol{b}$, Left, Firing rate modulation induced by running per area (colors) and in the model (gray; $\mathrm{CV}(\mathrm{E})=5 \%$ ), averaged across all periods of ongoing activity. Right, Fraction of neurons by area (colors) and in the model (gray; $\mathrm{CV}(\mathrm{E})=5 \%$ ) with significantly excited (positive bars) and inhibited (negative bars) responses to bouts of running (rank-sum test, ${ }^{*} p<0.005$ ). $c, A$ representative single-cell distribution of firing rates for rest (black) and running (brown) conditions. The overlaid distributions of firing rates are obtained by passing a standard normal distribution through the sigmoidal transfer function fit shown in the inset for rest (full gray line) and running (dashed gray line). The gain for each behavioral condition (orange lines) was estimated as the slope of the sigmoidal transfer function fit at the inflection point (see Materials and Methods). $\boldsymbol{d}$, Single-cell gain modulation ( $\Delta$ gain $=$ gain(running) - gain(rest)) by area (colors) and in the model (gray; $\mathrm{CV}(\mathrm{E})=5 \%)$ across all neurons during ongoing periods (bars represent $95 \% \mathrm{Cl}$; rank-sum test ${ }^{*} p<0.005$ ). e, Schematic of the network architecture, similar to Figure $2 a$, but with the sensory stimuli targeting subsets of both $E$ and I clusters, during both unperturbed and perturbed $(\delta \operatorname{var}(E))$ conditions. $f$, Average time course of the stimulus-decoding accuracy in the unperturbed (black) and perturbed (brown) conditions for the new stimulus input (notations as in Fig. 4c). $\boldsymbol{g}$, Time course of the mean stimulus-decoding accuracy across orientations during running and rest using neurons from V1 as predictors shows the anticipation of stimulus coding in the running condition (single sessions and session average, thin and thick lines, respectively; see Materials and Methods). $\boldsymbol{h}$, Decoding latency (first bin above chance decoding regions in $\boldsymbol{e}$ ) slows down along the anatomic hierarchy ( $x$ axis: anatomic hierarchy score from Siegle et al., 2019). Dotted (dashed) line with diamond $(" \mathrm{x}$ ") symbols show the latency during rest (running). $\boldsymbol{i}$, Difference in processing speed between running and resting (average latency of decoding accuracy between $40 \%$ and $80 \%$; $\boldsymbol{e}$, yellow area) reveals running-induced coding acceleration in all areas (colors) and in the model (gray; $\mathrm{CV}(\mathrm{E})=5 \%$ ). $t$ test, ${ }^{*} p<0.01$.

consistent with locomotion-induced effects in the visual cortical hierarchy. Increasing $\delta \operatorname{var}(\mathrm{I})$ left firing rates of I populations unchanged but led to a decrease of E population firing rates. This perturbation also induced mixed responses at the single-cell level, with a prevalence of inhibited responses in both populations. Finally, perturbations $\delta \mathrm{AMPA}$ and $\delta \mathrm{GABA}$ led to responses similar to those found when driving the mean $\mathrm{E}$ or I afferents, respectively.

Our theory thus suggests that it is possible to identify a specific perturbation by combining all its effects, including gain modulation, changes in stimulus processing speed, and singlecell physiological responses (Table 3).

\section{Changes in single-cell responses cannot explain the effects of perturbations on evoked activity}

Although we found that gain modulation captures the effects of perturbations on network activity, we investigate whether alternative explanations were also possible, in terms of traditional measures of stimulus responsiveness and selectivity.

We found that perturbations strongly affected the peak of single-cell responses to stimuli compared with baseline $(\triangle \mathrm{PSTH}$; Fig. $6 c$ ), as well as single-cell selectivity to stimuli with significant changes in their $d^{\prime}$ (Fig. $6 d$ ). We then tested whether perturbation-induced changes in stimulus responses or selectivity could explain the observed changes in stimulus processing speed. We first hypothesized that, if the response increase induced by the perturbation were larger for stimulus-selective compared with

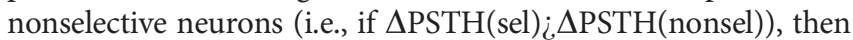
a perturbation increasing stimulus-responses could lead to faster stimulus processing speed. Likewise, we hypothesized that faster stimulus processing speed may be induced by perturbations improving single-cell selectivity $\left(d^{\prime}\right)$ to stimuli. Surprisingly, we found a complex relation between changes in single-cell responsiveness and selectivity to stimuli, induced by the perturbations, and modulation of stimulus processing speed (Fig. $6 c, d$ ). For perturbations targeting I populations $(\delta$ mean $(\mathrm{I}), \delta \operatorname{var}(\mathrm{I})$, and $\delta \mathrm{GABA})$, changes in responsiveness and selectivity were consistent with changes in processing speed $\left(R^{2}=0.92\right.$ and $R^{2}=0.70$ for responsiveness and selectivity, respectively). However, for perturbations targeting $\mathrm{E}$ populations $(\delta$ mean $(\mathrm{E}), \delta \operatorname{var}(\mathrm{E})$, and $\delta$ AMPA), changes in responsiveness and selectivity were not consistent with changes in processing speed $\left(R^{2}=0.05\right.$ and $R^{2}=$ 0.02 for responsiveness and selectivity, respectively). Strikingly, in the case of the perturbation $\delta \operatorname{var}(\mathrm{E})$, processing speed increased with larger perturbations, although responses and selectivity increasingly degraded. In the case of the perturbation $\delta$ mean(E) and $\delta$ AMPA, network performance likewise increased, but single-cell metrics where nonmonotonic in the 
value of the perturbation (Fig. $6 c, d$ ). Because changes in single-cell stimulus properties were only consistent with changes in processing speed for some perturbations $(\delta$ mean $(\mathrm{I}), \quad \delta \operatorname{var}(\mathrm{I}), \quad$ and $\delta$ GABA), but inconsistent for other perturbations, we thus conclude that they could not represent an alternative mechanism underlying the observed effects of perturbations.

Locomotion decreases single-cell gain and accelerates visual processing speed Our theory predicts a link between gain modulations measured during ongoing periods and changes in stimulus processing speed during evoked periods. We sought to experimentally test this prediction in freely running mice using electrophysiological recordings from the visual hierarchy, including the primary visual cortex (V1) and four higher cortical visual areas: $\mathrm{LM}, \mathrm{AL}, \mathrm{PM}$, and $\mathrm{AM}$ (open-source neuropixels dataset available from the Allen Institute) (Siegle et al., 2019). We interpreted periods where the animal was resting as akin to the "unperturbed" condition in our model, and periods where the animal was running as the "perturbed" condition (Fig. $7 a$ in the data). We thus set out to test our theory in the following three steps: (1) in each area, we estimated the effect of locomotion on single-cell responses and on intrinsic gain during ongoing periods; (2) based on these changes, we built a biologically plausible model of cortical circuits processing visual stimuli and predicted whether locomotion would accelerate or slow down visually evoked responses; and (3) we tested the prediction in each area with a decoding analysis of visually evoked population activity.

During periods of ongoing activity (in the absence of visual stimuli), we found that locomotion induced an overall increase in firing rate across all visual cortical areas (Fig. $7 b$, left), in agreement with previous studies (Niell and Stryker, 2010; Dipoppa et al., 2018; Stringer et al., 2019), although we found that locomotion led to complex responses inducing mixed excited and inhibited responses across neurons (Fig. $7 b$, right), as previously reported Dipoppa et al. (2018). We then estimated the single-cell transfer functions from spiking activity during ongoing periods both when the animal was at rest and in motion (Fig. 7c). We found that locomotion strongly modulated the single-cell gain in the absence of stimuli in all visual cortical areas (Fig. $7 d$ ). Specifically, we found that locomotion on average decreased the single-cell gain.

Our theory predicts that, in all visual cortical areas, the locomotion-induced increase in firing rates, the mixed excited and inhibited neural responses, and the decrease in intrinsic gain are consistent with a state-changing perturbation mediated by an increase in the variance of the input currents to $\mathrm{E}$ neurons ( $\delta$ var (E); Table 3). According to our theory, the decrease in gain leads to an acceleration of stimulus processing speed in all visual cortical areas.
We aimed at refining the model predictions on the locomotion effects on V1 and the visual cortical hierarchy by introducing a biologically plausible stimulation protocol in our spiking network. Following experimental evidence on anatomic connectivity in the visual pathway (Zhuang et al., 2013; Kloc and Maffei, 2014; Khan et al., 2018; Miska et al., 2018), we then modeled incoming visual stimuli as a transient increase in the input currents to both $\mathrm{E}$ and I neurons (Fig. 7e). We then modeled the effect of locomotion as an external perturbation inducing an increase in the variance of the inputs to $\mathrm{E}$ neurons $\delta \operatorname{var}(\mathrm{E})$, capturing the observed empirical effects of locomotion on ongoing periods in terms of gain decrease and mixed single-cell responses. In this model of visual processing, we found that locomotion accelerated visual processing speed during evoked period by $21 \pm 9 \mathrm{~ms}$ on average (mean \pm SD across 10 sessions; Fig. 7f). We thus set out to test this prediction in the empirical data.

Previous studies have observed an improvement in peak decoding performance during locomotion (Dadarlat and Stryker, 2017), but changes in decoding latency have not been investigated. To probe the speed and accuracy of visual responses in perturbed and unperturbed conditions, we performed a crossvalidated classification analysis to assess the amount of information regarding the orientation of drifting grating stimuli present in population spiking activity along the visual cortical hierarchy. Crucially, because decoding accuracy depends on sample size, we equalized number of trials between resting and running conditions. We found that trials in which the animal was running revealed both an increase in peak decoding accuracy and an anticipation of stimulus coding (shorter latency) compared with 
trials where the animal was stationary (Fig. $7 g$ ), consistently across the whole visual hierarchy (Fig. 7i). Furthermore, the time to reach significant decoding for each cortical area followed the anatomic hierarchy score in both unperturbed and perturbed conditions, consistent with the idea that information about the visual stimulus travels up a visual hierarchy in a feedforward fashion (Fig. 7h) (Siegle et al., 2019).

Given that locomotion induced an increase in firing rates in all cortical areas (Fig. 7b), we then examined the extent to which the observed effects of locomotion (increased peak accuracy and anticipation) were merely because of the increase in firing rates. We thus matched the distribution of firing rates between running and resting (Fig. 8; see Materials and Methods). We found that, after rate matching, the change in peak decoding accuracy decreased significantly (Fig. $8 d$ ). Crucially, the anticipation of stimulus processing speed induced by locomotion was still present in the rate-matched condition (Fig. $8 c$ ), confirming that it was independent of changes in firing rates. The same effect was preserved in the rate-matched model simulations as well (not shown). We thus concluded that the anticipation of visual processing speed induced by locomotion is consistent with a mechanism whereby locomotion decreases single-cell gain via an increase in the afferent variance $\delta \operatorname{var}(\mathrm{E})$ as predicted by our theory (Table 3).

\section{Discussion}

Cortical circuits flexibly adapt their information processing capabilities to changes in environmental demands and internal state. Empirical evidence suggests that these state-dependent modulations may occur already in the sensory cortex where they may be induced by top-down pathways or neuromodulation. Here, we presented a mechanistic theory explaining how stimulus processing speed can be regulated in a state-dependent manner via gain modulation, induced by transient changes in the afferent currents or in the strength of synaptic transmission.

Our theory entails a recurrent spiking network where excitatory and inhibitory neurons are arranged in clusters, generating metastable activity in the form of transient activation of subsets of clusters. We showed that gain modulation controls the timescale of metastable activity and thus the network's information processing speed and reaction times on stimulus presentation. Specifically, our theory predicted that perturbations that decrease (increase) the intrinsic single-cell gain during ongoing periods accelerate (slow down) the latency of stimulus responses.

We tested this prediction by examining the effect of locomotion on visual processing in freely running mice. We found that locomotion reduced the intrinsic single-cell gain during ongoing periods, thus accelerating stimulus-coding speed across the visual cortical hierarchy. Our theory suggests that the observed effects of locomotion are consistent with a perturbation that increases the spatial variance of the afferent currents to the local excitatory population. These results establish a new theory of state-dependent adaptation of cortical responses via gain modulation, unifying the effect of different pathways under a shared computational mechanism.

\section{Metastable activity in cortical circuits}

The crucial dynamical feature of our model is its metastable activity, whereby single-trial ensemble spike trains unfold through sequences of metastable states. States are long-lasting, with abrupt transitions between consecutive states. Metastable activity has been ubiquitously observed in a variety of cortical and subcortical areas, across species and tasks (Abeles et al., 1995; Jones et al., 2007; Ponce-Alvarez et al., 2012; Engel et al., 2016; Rich and Wallis, 2016; Sadacca et al., 2016; Maboudi et al., 2018; Taghia et al., 2018; Deco et al., 2019). Metastable activity can be used to predict behavior and was implicated as a neural substrate of cognitive function, such as attention (Engel et al., 2016), expectation (Mazzucato et al., 2019), and decision-making (Rich and Wallis, 2016; Taghia et al., 2018; Recanatesi et al., 2020). Metastable activity was observed also during ongoing periods, in the absence of sensory stimulation, suggesting that it may be an intrinsic dynamical regimen of cortical circuits (Mazzucato et al., 2015; Engel et al., 2016). Here, we showed how cortical circuits can flexibly adjust their performance and information processing speed via modulations of their metastable dynamics.

Metastable activity may naturally arise in circuits where multiple stable states, or attractors, are destabilized by external perturbations (Miller and Katz, 2010) or intrinsically generated variability (Deco and Hugues, 2012; Litwin-Kumar and Doiron, 2012; Mazzucato et al., 2015, 2019; Schaub et al., 2015; Recanatesi et al., 2020; Rostami et al., 2020). Biologically plausible models of metastable dynamics have been proposed in terms of recurrent spiking networks where neurons are arranged in clusters, reflecting the empirically observed assemblies of functionally correlated neurons (Song et al., 2005; Perin et al., 2011; Kiani et al., 2015; W. C. Lee et al., 2016). Clustered network models of metastable dynamics provide a parsimonious explanation of several physiological observations, such as stimulusinduced reductions of trial-to-trial variability (Churchland et al., 2010; Deco and Hugues, 2012; Litwin-Kumar and Doiron, 2014; Mazzucato et al., 2015; Rostami et al., 2020), of firing rate multistability (Mazzucato et al., 2015), and of neural dimensionality (Mazzucato et al., 2016). Compared with previous models of metastable dynamics, our results extend the biological plausibility of clustered networks in several aspects. The introduction of pairs of $\mathrm{E}$ and I clusters induces a tight balance where the $\mathrm{E}$ and I contributions to the postsynaptic currents of each neuron closely track each other with opposite signs (data not shown), as observed experimentally in cortical circuits (Okun and Lampl, 2008). Moreover, we showed that these networks operate in the inhibition stabilized regimen (Fig. 2c), which is believed to be the operational regimen of cortical circuits (Ozeki et al., 2009; Moore et al., 2018; Sanzeni et al., 2019). We showed that a heterogeneous distribution of cluster sizes naturally gives rise to lognormal distribution of firing rates (Fig. $2 b$, inset), as observed in cortical circuits (Shafi et al., 2007; Hromádka et al., 2008; O'Connor et al., 2010; Roxin et al., 2011). We then generalize the results in Mazzucato et al. (2019) to establish gain modulation as the general mechanism controlling that state-dependent changes in processing speed in recurrent circuits with metastable dynamics. This class of models thus provides a biologically plausible, mechanistic link between connectivity, dynamics, and information processing.

\section{Linking metastable activity to flexible cognitive function via gain modulation}

Recent studies have shown that cortical circuits may implement a variety of flexible cognitive computations by modulating the timescale of their intrinsic metastable dynamics (Mattia et al., 2013; Engel et al., 2016; Rich and Wallis, 2016; Deco et al., 2019; Mazzucato et al., 2019). Our results establish a comprehensive framework to investigate the extent of this hypothesis. We propose that gain modulation is the neural mechanism underlying flexible state-dependent cortical computation. Specifically, we 
showed that gain modulation controls the timescale of metastable dynamics, which, in turn, determines the network's information processing speed.

Our theoretical framework to link gain modulation to changes in potential barrier heights is based on the effective mean field theory (Mascaro and Amit, 1999; Mattia et al., 2013; Mazzucato et al., 2019), which we used to reduce a multidimensional system to obtain an effective potential describing a single population. Although this approach is exact in the case of networks with symmetric connectivity, it represents only an approximation to the full network dynamics in the case of networks with asymmetric couplings, such as the ones considered in this study (Rodríguez-Sánchez et al., 2020). It would be interesting to extend our results to an exact framework by estimating the network Lyapunov function (Yan et al., 2013).

\section{Alternative models of gain modulation}

Previous studies have suggested gain modulation as a mechanism to sharpen single-cell tuning curves without affecting selectivity (Cardin et al., 2008; Haider and McCormick, 2009), potentially mediating alertness (Cano et al., 2006) or attention (McAdams and Maunsell, 1999; Treue and Trujillo, 1999; Rabinowitz et al., 2015). In those studies, gain modulation was defined as change in the single-neuron response function to stimuli of increasing contrast. Here, we have taken a different approach and defined gain as the slope of the intrinsic neuronal current-to-rate function during ongoing periods (i.e., in the absence of stimuli) (see also Chance et al., 2002; Haider and McCormick, 2009; Mazzucato et al., 2019), as opposed to the contrast response function. We have classified mechanisms of gain modulation which act by changing the mean or spatial variance across neurons of the cell type-specific afferent currents to the local cortical circuit, where we modeled afferent currents as constant biases; or by changing the recurrent couplings. The rationale for our choice was to investigate the effects on internally generated variability in a network whose dynamics were entirely deterministic. Alternatively, one could model external currents as time-dependent inputs with fast noise, such as Poisson processes or colored noise. In that case, changes in background noise because of barrages of synaptic inputs are capable of inducing gain modulation as well (Chance et al., 2002; Haider and McCormick, 2009). Previous work compared these different kinds of perturbations (Poisson noise or afferent spatial variance) in the case of the perturbation $\delta \operatorname{var}(\mathrm{E})$ (Mazzucato et al., 2019), showing that they may lead to similar outcomes.

\section{Physiologic mechanisms of gain modulation}

Several different physiological pathways can modulate the gain of the intrinsic neuronal transfer function, including neuromodulation, top-down, and corticocortical interactions. Gain modulation can also be induced artificially by means of optogenetic or pharmacological manipulations. The perturbations investigated in our model may be related to different pathways and implicated in various types of cognitive function.

\section{Neuromodulation}

Neuromodulatory pathways strongly affect sensory processing in cortical circuits by changing cell type-specific afferent currents to the circuit, in some cases controlling their dynamical regimen (McGinley et al., 2015). Our theory may be applicable to explain the effects of cholinergic and serotonergic activation on sensory cortex.

Cholinergic pathways, modulating ionic currents in pyramidal cells (McCormick, 1992), can control cortical states and mediate the effects of arousal and locomotion. Artificial stimulation of cholinergic pathways was shown to improve sensory coding in visual (Goard and Dan, 2009; Pinto et al., 2013) and barrel cortex (Eggermann et al., 2014). Cholinergic stimulation alone in the absence of sensory stimuli was shown to induce mixed responses with different neural populations increasing or decreasing their spiking activity (Goard and Dan, 2009). Our theory shows that these combined experimental observations (coding improvement and mixed firing rate changes) are consistent with a mechanism whereby cholinergic activation induces an increase in $\delta \operatorname{var}(\mathrm{E})$ afferents to sensory cortex, inducing an acceleration of sensory processing (Fig. 7).

Activation of serotonergic pathways by stimulation of dorsal raphe serotonergic neurons or local iontophoresis was shown to transiently degrade stimulus coding in sensory cortex, decreasing responses to mechanosensory stimuli (Dugué et al., 2014) and increasing the latency of the first spike evoked by auditory stimuli (Hurley et al., 2002). Serotonergic stimulation was shown to decrease firing rates in the olfactory cortex (Lottem et al., 2016), inferior colliculus (Hurley et al., 2002), and primary visual cortex (Seillier et al., 2017; Michaiel et al., 2019). Our theory shows that these experimental observations (coding degradation and decreased firing rates) are consistent with two alternative mechanisms: either an increase in the afferent currents to I populations (i.e., $\delta$ mean $(\mathrm{I})>0$ ) implementing the paradoxical inhibition effect (Tsodyks et al., 1997) or a decrease in the afferents to $\mathrm{E}$ populations (i.e., $\delta$ mean $(\mathrm{E})<0$ ). Future experiments could test between these two alternatives.

\section{Top-down projections}

A prominent feature of sensory cortex is the integration of feedforward and corticocortical feedback pathways at each stage of sensory processing (Felleman and Van Essen, 1991). In particular, top-down projections from higher cortical areas to sensory cortex are known to modulate the speed and accuracy of sensory processing (Mazzucato et al., 2019). Our theory may explain the effects of activating several corticocortical pathways.

Activation of feedback axons from motor cortex (M1) to somatosensory cortex (S1) was shown to increase activity in S1 during whisking (Petreanu et al., 2012) and led to faster and more accurate responses to whisker stimulation (Zagha et al., 2013). Suppression of the same pathway induced slower S1 responses to whisking in awake mice. Our theory shows that the effect of these corticocortical perturbations is consistent with an increase in the mean afferent currents to E populations in S1 (i. e., the $\delta$ mean(E) perturbation), leading to higher firing rates and faster processing speed.

Expectation and arousal are known to strongly modulate neural activity in sensory cortices (Salkoff et al., 2020). Expected stimuli are processed faster and more accurately than unexpected stimuli both in auditory (Jaramillo and Zador, 2011) and gustatory cortex (Samuelsen et al., 2012). Experimental evidence shows that the anticipation of sensory processing induced by expectation is mediated by top-down projections from the amygdala to the gustatory cortex (Samuelsen et al., 2012), whose activation elicits mixed excited and inhibited responses in both pyramidal and inhibitory cells in the gustatory cortex (Samuelsen et al., 2012; Vincis and Fontanini, 2016). Our model shows that, while an acceleration of stimulus processing speed may in principle be mediated by different state-changing perturbations, only the $\delta \operatorname{var}(\mathrm{E})$ perturbation is consistent with the empirically observed mixed responses. Indeed, our theory suggests that these top-down projections may operate by inducing an increase in the spatial variance of the afferent currents to the 
E population ( $\delta \operatorname{var}(\mathrm{E})$ perturbation, extending previous results in Mazzucato et al. (2019) to networks including inhibitory clusters).

In attentional tasks, distractors slow down reaction times (Grueninger and Pribram, 1969; Treisman and Gelade, 1980), a behavioral effect that may be mediated by changes in the speed and accuracy of sensory processing in cortical circuits (Desimone and Duncan, 1995). The presence of distracting stimuli within a neurons receptive field suppresses its responses to the preferred stimulus (Knierim and Van Essen, 1992). The underlying mechanism may recruit lateral inhibition onto the local cortical circuit (Reynolds and Heeger, 2009; Gilbert and Li, 2013). Our theory shows that this mechanism is consistent with a modulation of the afferents to local I populations, mediated by either an increase in $\delta$ mean(I) or $\delta \operatorname{var}(\mathrm{I})$. It would be interesting to discriminate between these two perturbations with future experiments.

\section{Optogenetic and pharmacological manipulations}

Our theory may shed light on the effects of manipulation experiments. Optogenetic activation (inactivation) of specific E or I cells (Arenkiel et al., 2007; Li et al., 2019) has been modeled as an increase (decrease) of the afferent currents to those cells (Ebsch and Rosenbaum, 2018; Sanzeni et al., 2019; Mahrach et al., 2020). However, protein expression may not be complete across all cells of the targeted population; and even in the case of complete expression across the targeted population, different cells may be more or less sensitive to laser stimulation. Thus, the effect of optogenetic stimulation on the targeted population may then be more accurately modeled by a concurrent change in both mean and variance of the targeted cell type-specific afferents (e.g., $\delta$ mean(E) and $\delta \operatorname{var}(\mathrm{E})$ for E populations; $\delta$ mean(I) and $\delta \operatorname{var}(\mathrm{I})$ for I populations). Recent studies showed that, while a homogeneous stimulation of all I cell types simultaneously can be captured by a model of E-I recurrently coupled neurons (as in our model), partial activation of specific inhibitory cell types may induce more complex responses (Otchy et al., 2015; Phillips and Hasenstaub, 2016; Li et al., 2019; Sanzeni et al., 2019; Mahrach et al., 2020). We plan to revisit this issue in the future.

Our theory may also be applicable to the effects of pharmacological manipulations of different synaptic receptors. In particular, the effects of combined local injection of AMPA/kainate and NMDA receptor antagonists (agonists) may be recapitulated by a decrease (increase) in $\delta$ AMPA, which correspondingly perturb the value of $J_{I E}, J_{E E}$ couplings. Similarly, the effects of local injection of GABA receptor antagonists (agonists) may be recapitulated by a decrease (increase) in $\delta \mathrm{GABA}$, which correspondingly perturb the value of $J_{E I}$, $J_{I I}$ couplings.

\section{Locomotion and gain modulation}

Locomotion has been shown to modulate visually evoked activity (Niell and Stryker, 2010) and is sufficient in driving activity in mouse V1 (Saleem et al., 2013; Leinweber et al., 2017). Our results were consistent with previous studies in showing that locomotion affects the activity of neurons in the visual cortical hierarchy during both ongoing and stimulus-evoked activity. We found that locomotion in the absence of sensory stimuli induces an average increase in firing rates. At the single-cell level, we reported a complex mix of excited and inhibited responses in both $\mathrm{E}$ and I cells, also consistent with previous results (Fu et al., 2014; Dipoppa et al., 2018). Crucially, we uncovered that locomotion decreased the single-cell gain during ongoing activity across the board in the visual cortical hierarchy (Fig. $7 d$ ). Our theory predicted that the observed decrease in gain would lead to an acceleration of visual processing during locomotion in cortex. This prediction was confirmed in the data (Fig. 7i). The acceleration of processing speed observed in cortex did not depend on the locomotion-induced changes in firing rates and was still present even after matching the firing rate distributions between running and rest conditions (Fig. 8). Our model of the perturbation effects induced by locomotion (increased firing rates with mixed excited and inhibited responses, and faster visual processing) suggests that the effect of locomotion may be mediated by an increase in the spatial variance of the afferent current to the $\mathrm{E}$ populations ( $\delta \operatorname{var}(\mathrm{E})$ perturbation) (Niell and Stryker, 2010; Ayaz et al., 2013; Fu et al., 2014). Concretely, gain modulation may be implemented via the combined effect of activating neuromodulatory pathways, such as cholinergic (Fu et al., 2014) and noradrenergic (Polack et al., 2013) inputs.

\section{References}

Abeles M, Bergman H, Gat I, Meilijson I, Seidemann E, Tishby N, Vaadia E (1995) Cortical activity flips among quasi-stationary states. Proc Natl Acad Sci USA 92:8616-8620.

Amit DJ, Brunel N (1997) Model of global spontaneous activity and local structured activity during delay periods in the cerebral cortex. Cereb Cortex 7:237-252.

Arenkiel BR, Peca J, Davison IG, Feliciano C, Deisseroth K, Augustine GJ, Ehlers MD, Feng G (2007) In vivo light-induced activation of neural circuitry in transgenic mice expressing channelrhodopsin-2. Neuron 54:205-218.

Ayaz A, Saleem AB, Schölvinck ML, Carandini M (2013) Locomotion controls spatial integration in mouse visual cortex. Curr Biol 23:890-894.

Cano M, Bezdudnaya T, Swadlow HA, Alonso JM (2006) Brain state and contrast sensitivity in the awake visual thalamus. Nat Neurosci 9:12401242.

Cardin JA, Palmer LA, Contreras D (2008) Cellular mechanisms underlying stimulus-dependent gain modulation in primary visual cortex neurons in vivo. Neuron 59:150-160.

Chance FS, Abbott LF, Reyes AD (2002) Gain modulation from background synaptic input. Neuron 35:773-782.

Chen TW, Li N, Daie K, Svoboda K (2017) A map of anticipatory activity in mouse motor cortex. Neuron 94:866-879.

Churchland MM, Yu BM, Cunningham JP, Sugrue LP, Cohen MR, Corrado GS, Newsome WT, Clark AM, Hosseini P, Scott BB, Bradley DC, Smith MA, Kohn A, Movshon JA, Armstrong KM, Moore T, Chang SW, Snyder LH, Lisberger SG, Priebe NJ, et al. (2010) Stimulus onset quenches neural variability: a widespread cortical phenomenon. Nat Neurosci 13: 369-378

Cohen MR, Maunsell JH (2009) Attention improves performance primarily by reducing interneuronal correlations. Nat Neurosci 12:1594-1600.

Dadarlat MC, Stryker MP (2017) Locomotion enhances neural encoding of visual stimuli in mouse v1. J Neurosci 37:3764-3775.

Deco G, Hugues E (2012) Neural network mechanisms underlying stimulus driven variability reduction. PLoS Comput Biol 8:e1002395.

Deco G, Cruzat J, Cabral J, Tagliazucchi E, Laufs H, Logothetis NK, Kringelbach ML (2019) Awakening: predicting external stimulation to force transitions between different brain states. Proc Natl Acad Sci USA 116:18088-18097.

Desimone R, Duncan J (1995) Neural mechanisms of selective visual attention. Annu Rev Neurosci 18:193-222.

Dipoppa M, Ranson A, Krumin M, Pachitariu M, Carandini M, Harris KD (2018) Vision and locomotion shape the interactions between neuron types in mouse visual cortex. Neuron 98:602-615.

Dugué GP, Lörincz ML, Lottem E, Audero E, Matias S, Correia PA, Léna C, Mainen ZF (2014) Optogenetic recruitment of dorsal raphe serotonergic neurons acutely decreases mechanosensory responsivity in behaving mice. PLoS One 9:e105941.

Ebsch C, Rosenbaum R (2018) Imbalanced amplification: a mechanism of amplification and suppression from local imbalance of excitation and inhibition in cortical circuits. PLoS Comput Biol 14:e1006048. 
Eggermann E, Kremer Y, Crochet S, Petersen CC (2014) Cholinergic signals in mouse barrel cortex during active whisker sensing. Cell Rep 9:16541660.

Engel TA, Steinmetz NA, Gieselmann MA, Thiele A, Moore T, Boahen K (2016) Selective modulation of cortical state during spatial attention. Science 354:1140-1144.

Felleman DJ, Van Essen DC (1991) Distributed hierarchical processing in the primate cerebral cortex. Cereb Cortex 1:1-47.

Fourcaud N, Brunel N (2002) Dynamics of the firing probability of noisy integrate-and-fire neurons. Neural Comput 14:2057-2110.

Fu Y, Tucciarone JM, Espinosa JS, Sheng N, Darcy DP, Nicoll RA, Huang ZJ, Stryker MP (2014) A cortical circuit for gain control by behavioral state. Cell 156:1139-1152.

Gilbert CD, Li W (2013) Top-down influences on visual processing. Nat Rev Neurosci 14:350-363.

Goard M, Dan Y (2009) Basal forebrain activation enhances cortical coding of natural scenes. Nat Neurosci 12:1444-1449.

Grueninger WE, Pribram KH (1969) Effects of spatial and nonspatial distractors on performance latency of monkeys with frontal lesions. J Comp Physiol Psychol 68:203-209.

Guo ZV, Li N, Huber D, Ophir E, Gutnisky D, Ting JT, Feng G, Svoboda K (2014) Flow of cortical activity underlying a tactile decision in mice. Neuron 81:179-194.

Haider B, McCormick DA (2009) Rapid neocortical dynamics: cellular and network mechanisms. Neuron 62:171-189.

Hänggi P, Talkner P, Borkovec M (1990) Reaction-rate theory: fifty years after Kramers. Rev Mod Phys 62:251-341.

Hromádka T, DeWeese MR, Zador AM (2008) Sparse representation of sounds in the unanesthetized auditory cortex. PLoS Biol 6:e16.

Huang C, Ruff DA, Pyle R, Rosenbaum R, Cohen MR, Doiron B (2019) Circuit models of low-dimensional shared variability in cortical networks. Neuron 101:337-348.

Hurley LM, Thompson AM, Pollak GD (2002) Serotonin in the inferior colliculus. Hear Res 168:1-11.

Jaramillo S, Zador A (2011) Auditory cortex mediates the perceptual effects of acoustic temporal expectation. Nat Neurosci 14:246-251.

Jezzini A, Mazzucato L, La Camera G, Fontanini A (2013) Processing of hedonic and chemosensory features of taste in medial prefrontal and insular networks. J Neurosci 33:18966-18978.

Jones LM, Fontanini A, Sadacca BF, Miller P, Katz DB (2007) Natural stimuli evoke dynamic sequences of states in sensory cortical ensembles. Proc Natl Acad Sci USA 104:18772-18777.

Khan AG, Poort J, Chadwick A, Blot A, Sahani M, Mrsic-Flogel TD, Hofer SB (2018) Distinct learning-induced changes in stimulus selectivity and interactions of GABAergic interneuron classes in visual cortex. Nat Neurosci 21:851-859.

Kiani R, Cueva CJ, Reppas JB, Peixoto D, Ryu SI, Newsome WT (2015) Natural grouping of neural responses reveals spatially segregated clusters in prearcuate cortex. Neuron 85:1359-1373.

Kloc M, Maffei A (2014) Target-specific properties of thalamocortical synapses onto layer 4 of mouse primary visual cortex. J Neurosci 34:1545515465.

Knierim JJ, Van Essen DC (1992) Neuronal responses to static texture patterns in area v1 of the alert macaque monkey. J Neurophysiol 67:961980.

Lee AM, Hoy JL, Bonci A, Wilbrecht L, Stryker MP, Niell CM (2014) Identification of a brainstem circuit regulating visual cortical state in parallel with locomotion. Neuron 83:455-466.

Lee WC, Bonin V, Reed M, Graham BJ, Hood G, Glattfelder K, Reid RC (2016) Anatomy and function of an excitatory network in the visual cortex. Nature 532:370-374.

Leinweber M, Ward DR, Sobczak JM, Attinger A, Keller GB (2017) A sensorimotor circuit in mouse cortex for visual flow predictions. Neuron 95:1420-1432.

Li N, Chen S, Guo ZV, Chen H, Huo Y, Inagaki HK, Chen G, Davis C, Hansel D, Guo C, Svoboda K (2019) Spatiotemporal constraints on optogenetic inactivation in cortical circuits. Elife 8:e48622.

Lim S, McKee JL, Woloszyn L, Amit Y, Freedman DJ, Sheinberg DL, Brunel $\mathrm{N}$ (2015) Inferring learning rules from distributions of firing rates in cortical neurons. Nat Neurosci 18:1804-1810.
Litwin-Kumar A, Doiron B (2012) Slow dynamics and high variability in balanced cortical networks with clustered connections. Nat Neurosci 15:1498-1505

Litwin-Kumar A, Doiron B (2014) Formation and maintenance of neuronal assemblies through synaptic plasticity. Nat Commun 5:5319.

Lottem E, Lörincz ML, Mainen ZF (2016) Optogenetic activation of dorsal raphe serotonin neurons rapidly inhibits spontaneous but not odorevoked activity in olfactory cortex. J Neurosci 36:7-18

Maboudi K, Ackermann E, de Jong LW, Pfeiffer BE, Foster D, Diba K, Kemere C (2018) Uncovering temporal structure in hippocampal output patterns. Elife 7:e34467.

Mahrach A, Chen G, Li N, van Vreeswijk C, Hansel D (2020) Mechanisms underlying the response of mouse cortical networks to optogenetic manipulation. Elife 9:e49967.

Mascaro M, Amit DJ (1999) Effective neural response function for collective population states. Network 10:351-373.

Mattia M, Sanchez-Vives MV (2012) Exploring the spectrum of dynamical regimes and timescales in spontaneous cortical activity. Cogn Neurodyn 6:239-250.

Mattia M, Pani P, Mirabella G, Costa S, Del Giudice P, Ferraina S (2013) Heterogeneous attractor cell assemblies for motor planning in premotor cortex. J Neurosci 33:11155-11168.

Mazzucato L, Fontanini A, La Camera G (2015) Dynamics of multistable states during ongoing and evoked cortical activity. J Neurosci 35:82148231 .

Mazzucato L, Fontanini A, La Camera G (2016) Stimuli reduce the dimensionality of cortical activity. Front Syst Neurosci 10:11.

Mazzucato L, La Camera G, Fontanini A (2019) Expectation-induced modulation of metastable activity underlies faster coding of sensory stimuli. Nat Neurosci 22:787-796.

McAdams CJ, Maunsell JH (1999) Effects of attention on orientation-tuning functions of single neurons in macaque cortical area v4. J Neurosci 19:431-441.

McCormick DA (1992) Neurotransmitter actions in the thalamus and cerebral cortex and their role in neuromodulation of thalamocortical activity. Prog Neurobiol 39:337-388.

McGinley MJ, Vinck M, Reimer J, Batista-Brito R, Zagha E, Cadwell CR, Tolias AS, Cardin JA, McCormick DA (2015) Waking state: rapid variations modulate neural and behavioral responses. Neuron 87:1143-1161.

Michaiel AM, Parker PR, Niell CM (2019) A hallucinogenic serotonin-2a receptor agonist reduces visual response gain and alters temporal dynamics in mouse v1. Cell Rep 26:3475-3483.

Miller P, Katz DB (2010) Stochastic transitions between neural states in taste processing and decision-making. J Neurosci 30:2559-2570.

Miska NJ, Richter LM, Cary BA, Gjorgjieva J, Turrigiano GG (2018) Sensory experience inversely regulates feedforward and feedback excitation-inhibition ratio in rodent visual cortex. Elife 7:e38846.

Moore AK, Weible AP, Balmer TS, Trussell LO, Wehr M (2018) Rapid rebalancing of excitation and inhibition by cortical circuitry. Neuron 97:1341-1355

Nelson A, Schneider DM, Takatoh J, Sakurai K, Wang F, Mooney R (2013) A circuit for motor cortical modulation of auditory cortical activity. J Neurosci 33:14342-14353.

Niell CM, Stryker MP (2010) Modulation of visual responses by behavioral state in mouse visual cortex. Neuron 65:472-479.

Niemi P, Näätänen R (1981) Foreperiod and simple reaction time. Psychol Bull 89:133-162.

O'Connor DH, Peron SP, Huber D, Svoboda K (2010) Neural activity in barrel cortex underlying vibrissa-based object localization in mice. Neuron 67:1048-1061.

Okun M, Lampl I (2008) Instantaneous correlation of excitation and inhibition during ongoing and sensory-evoked activities. Nat Neurosci 11:535537.

Otchy TM, Wolff SB, Rhee JY, Pehlevan C, Kawai R, Kempf A, Gobes SM, Ölveczky BP (2015) Acute off-target effects of neural circuit manipulations. Nature 528:358-363.

Ozeki H, Finn IM, Schaffer ES, Miller KD, Ferster D (2009) Inhibitory stabilization of the cortical network underlies visual surround suppression. Neuron 62:578-592.

Pereira U, Brunel N (2018) Attractor dynamics in networks with learning rules inferred from in vivo data. Neuron 99:227-238. 
Perin R, Berger TK, Markram H (2011) A synaptic organizing principle for cortical neuronal groups. Proc Natl Acad Sci USA 108:5419-5424.

Petreanu L, Gutnisky DA, Huber D, Xu Nl, O'Connor DH, Tian L, Looger L, Svoboda K (2012) Activity in motor-sensory projections reveals distributed coding in somatosensation. Nature 489:299-303.

Phillips EA, Hasenstaub AR (2016) Asymmetric effects of activating and inactivating cortical interneurons. Elife 5:e18383.

Pinto L, Goard MJ, Estandian D, Xu M, Kwan AC, Lee SH, Harrison TC, Feng G, Dan Y (2013) Fast modulation of visual perception by basal forebrain cholinergic neurons. Nat Neurosci 16:1857-1863.

Polack PO, Friedman J, Golshani P (2013) Cellular mechanisms of brain state-dependent gain modulation in visual cortex. Nat Neurosci 16:1331-1339.

Ponce-Alvarez A, Nacher V, Luna R, Riehle A, Romo R (2012) Dynamics of cortical neuronal ensembles transit from decision making to storage for later report. J Neurosci 32:11956-11969.

Rabinowitz NC, Goris RL, Cohen M, Simoncelli EP (2015) Attention stabilizes the shared gain of V4 populations. Elife 4:e08998.

Recanatesi S, Pereira U, Murakami M, Mainen Z, Mazzucato L (2020) Metastable attractors explain the variable timing of stable behavioral action sequences. bioRxiv

Reimer J, McGinley MJ, Liu Y, Rodenkirch C, Wang Q, McCormick DA, Tolias AS (2016) Pupil fluctuations track rapid changes in adrenergic and cholinergic activity in cortex. Nat Commun 7:13289-13287.

Reynolds JH, Heeger DJ (2009) The normalization model of attention. Neuron 61:168-185.

Rich EL, Wallis JD (2016) Decoding subjective decisions from orbitofrontal cortex. Nat Neurosci 19:973-980.

Rodríguez-Sánchez P, Van Nes EH, Scheffer M (2020) Climbing Escher's stairs: a way to approximate stability landscapes in multidimensional systems. PLoS Comput Biol 16:e1007788.

Rostami V, Rost T, Riehle A, van Albada SJ, Nawrot MP (2020) Spiking neural network model of motor cortex with joint excitatory and inhibitory clusters reflects task uncertainty, reaction times, and variability dynamics. bioRxiv. doi: 10.1101/2020.02.27.968339.

Roxin A, Brunel N, Hansel D, Mongillo G, van Vreeswiik C (2011) On the distribution of firing rates in networks of cortical neurons. J Neurosci 31:16217-16226.

Sadacca BF, Mukherjee N, Vladusich T, Li JX, Katz DB, Miller P (2016) The behavioral relevance of cortical neural ensemble responses emerges suddenly. J Neurosci 36:655-669.

Saleem AB, Ayaz A, Jeffery KJ, Harris KD, Carandini M (2013) Integration of visual motion and locomotion in mouse visual cortex. Nat Neurosci 16:1864-1869.

Salkoff DB, Zagha E, McCarthy E, McCormick DA (2020) Movement and performance explain widespread cortical activity in a visual detection task. Cereb Cortex 30:421-437.

Samuelsen CL, Gardner MP, Fontanini A (2012) Effects of cue-triggered expectation on cortical processing of taste. Neuron 74:410-422.
Sanzeni A, Akitake B, Goldbach HC, Leedy CE, Brunel N, Histed MH (2019) Inhibition stabilization is a widespread property of cortical networks. bioRxiv. doi: 10.1101/656710.

Schaub MT, Billeh YN, Anastassiou CA, Koch C, Barahona M (2015) Emergence of slow-switching assemblies in structured neuronal networks. PLoS Comput Biol 11:e1004196.

Seillier L, Lorenz C, Kawaguchi K, Ott T, Nieder A, Pourriahi P, Nienborg H (2017) Serotonin decreases the gain of visual responses in awake macaque v1. J Neurosci 37:11390-11405.

Shafi M, Zhou Y, Quintana J, Chow C, Fuster J, Bodner M (2007) Variability in neuronal activity in primate cortex during working memory tasks. Neuroscience 146:1082-1108

Siegle JH, Jia X, Durand S, Gale S, Bennett C, Graddis N, Heller G, Ramirez TK, Choi H, Luviano JA (2019) A survey of spiking activity reveals a functional hierarchy of mouse corticothalamic visual areas. bioRxiv. doi: 10.1101/ 805010 .

Song S, Sjöström PJ, Reigl M, Nelson S, Chklovskii DB (2005) Highly nonrandom features of synaptic connectivity in local cortical circuits. PLoS Biol 3:e68.

Stringer C, Pachitariu M, Steinmetz N, Carandini M, Harris KD (2019) High-dimensional geometry of population responses in visual cortex. Nature 571:361-365.

Taghia J, Cai W, Ryali S, Kochalka J, Nicholas J, Chen T, Menon V (2018) Uncovering hidden brain state dynamics that regulate performance and decision-making during cognition. Nat Commun 9:2505.

Treisman AM, Gelade G (1980) A feature-integration theory of attention. Cogn Psychol 12:97-136.

Treue S, Trujillo JCM (1999) Feature-based attention influences motion processing gain in macaque visual cortex. Nature 399:575-579.

Tsodyks MV, Skaggs WE, Sejnowski TJ, McNaughton BL (1997) Paradoxical effects of external modulation of inhibitory interneurons. J Neurosci 17:4382-4388.

Vincis R, Fontanini A (2016) Associative learning changes cross-modal representations in the gustatory cortex. Elife 5:e16420.

Yan H, Zhao L, Hu L, Wang X, Wang E, Wang J (2013) Nonequilibrium landscape theory of neural networks. Proc Natl Acad Sci USA 110: E4185-E4194.

Zagha E, Casale AE, Sachdev RN, McGinley MJ, McCormick DA (2013) Motor cortex feedback influences sensory processing by modulating network state. Neuron 79:567-578.

Zagha E, Ge X, McCormick DA (2015) Competing neural ensembles in motor cortex gate goal-directed motor output. Neuron 88:565-577.

Zhang S, Xu M, Kamigaki T, Do JP, Chang WC, Jenvay S, Miyamichi K, Luo L, Dan Y (2014) Long-range and local circuits for top-down modulation of visual cortex processing. Science 345:660-665.

Zhuang J, Stoelzel CR, Bereshpolova Y, Huff JM, Hei X, Alonso JM, Swadlow HA (2013) Layer 4 in primary visual cortex of the awake rabbit: contrasting properties of simple cells and putative feedforward inhibitory interneurons. J Neurosci 33:11372-11389. 